\title{
Dielectronic recombination of argon-like ions
}

\author{
D. Nikolić ${ }^{1}$, T. W. Gorczyca ${ }^{1}$, K. T. Korista ${ }^{1}$, and N. R. Badnell ${ }^{2}$ \\ 1 Department of Physics, Western Michigan University, Kalamazoo, MI 49008, USA \\ e-mail: gorczyca@wich.edu \\ 2 Department of Physics, University of Strathclyde, Glasgow G4 0NG, UK
}

Received 23 March 2010 / Accepted 16 April 2010

\begin{abstract}
Context. We present a theoretical investigation of dielectronic recombination (DR) of Ar-like ions that sheds new light on the behavior of the rate coefficient at low-temperatures where these ions form in photoionized plasmas.

Aims. We provide results for the total and partial Maxwellian-averaged DR rate coefficients from the initial ground level of K II$\mathrm{Zn}$ XIII ions. It is expected that these new results will advance the accuracy of the ionization balance for Ar-like M-shell ions and pave the way towards a detailed modeling of astrophysically relevant X-ray absorption features.

Methods. We utilize the AUTOSTRUCTURE computer code to obtain the accurate core-excitation thresholds in target ions and carry out multiconfiguration Breit-Pauli (MCBP) calculations of the DR cross section in the independent-processes, isolated-resonance, distorted-wave (IPIRDW) approximation.

Results. Our results mediate the complete absence of direct DR calculations for certain Ar-like ions and question the reliability of the existing empirical rate formulas, often inferred from renormalized data within this isoelectronic sequence.
\end{abstract}

Key words. atomic data - atomic processes - plasmas - scattering

\section{Introduction}

Atomic structure and dynamic behavior of highly-charged ions is one of the key ingredients presently required for both laboratory plasma diagnostics and interpretation of astrophysical phenomena (Fawcett 1991; Liedhal 2000; Kallman \& Palmeri 2007). The most common diagnostic technique to probe for electron temperature of laser-produced, fusion, or astrophysical plasmas involves spectroscopical observations of intensity ratios of EUV or X-ray emission lines coming from consecutive ionization stages of a single plasma component. The accuracy of derived plasma parameters is strongly affected by uncertainties in chemical abundances, often inherited through the use of unreliable collisional ionization and/or dielectronic recombination (DR) rates (Savin \& Laming 2002). Seon et al. (2003) investigated the effect of the uncertainties in DR rates on an isoelectronic line ratio in $\mathrm{Ti}$ and $\mathrm{Cr}$ plasmas and found substantial differences in the fractional abundances obtained for $\mathrm{Ti} \mathrm{V}$ and $\mathrm{Cr}$ VII ions (a shift of the curves to lower temperatures) as compared to those inferred using the recommended recombination rate coefficients (Mazzotta et al. 1998).

In recent years an enormous amount of progress, both theoretically and experimentally, has been made in improving the DR rate database along isoelectronic series within the first and second rows, and the third row up through Mg-like ions (see the review by Kallman \& Palmeri 2007, and references therein). In most cases it is found that the newly determined DR rates are significantly larger than their earlier recommended estimates, having profound consequences on the ionization balance and thermal equilibrium in both photoionized and collisionally ionized plasmas, from the solar corona to Active Galactic Nuclei (AGN) (see, for example, Chakravorty et al. 2008, 2009; Bryans et al. 2006, 2009a,b; Dere et al. 2009). In this work, we present improved theoretical predictions of the DR rates of Ar-like ions. Of these ions, those of the iron peak elements are some of the more abundant in cosmic plasmas. In much of the remainder of this introduction we will provide some of the motivation in improving their atomic database, in particular their DR rate coefficients.

The strong contribution of M-shell Fe ions to the unresolved transition array of inner-shell absorption lines in $\sim 15-17 \AA$ X-ray spectra of several AGN observed with XMM-Newton and Chandra, was initially not well understood (Netzer 2004). The fact that AGN photoionization models initially overpredicted the average ionization stage of iron was attributed in part to an underestimate in the low-temperature DR rate coefficients for M-shell iron (Kraemer et al. 2004), and constituted the main motivation behind the benchmark calculations recently performed by Badnell (2006), as well as experimental and theoretical results presented in Lukić et al. (2007). The present work further extends the calculations of Badnell (2006) for Fe IX ions by augmenting the configuration interaction $(\mathrm{CI})$ with some of the most important $\Delta n_{\mathrm{c}}=0$ ionic core excitations (Aggarwal et al. 2006; Zeng et al. 2006).

In the framework of testing nucleosynthesis models, Ellison et al. (2001) identified Co as a rewarding element to study galactic and stellar formation histories through the observed abundance trends (del Peloso et al. 2005). For example, the agreement of the modeled time-dependent ejecta compositions and velocities with observed Type 1a supernovae spectra requires a substantial initial presence of $\mathrm{Ni}, \mathrm{Co}$, and $\mathrm{Fe}$ in the outer layers of the ejecta (Hillebrandt \& Niemeyer 2000). In the present work, we study the photorecombination of CoX as an example of a 
heavier iron peak element for which the stellar photospheric elemental abundances are less well known (Adelman et al. 2000).

Nickel is one of the most important heavy impurities in tokamaks and early attempts by the TFR Group (1980) to model the fractional abundance of its charge states suffered from deficient DR data. Recent simulations of the observed plasma emission from magnetic confinement fusion devices, namely the JET tokamak in Abingdon and RFX in Padova (Mattioli et al. 2004), also lacked accurate recombination rate coefficients for the Ni XI ion. However, reliable electron impact ionization data of the remaining ions in the nickel isonuclear sequence have been provided by Pindzola et al. (1991) and were widely used by Mattioli et al. (2004) in simulations of Ni emission line spectra. The past few years have marked a renewed theoretical interest (Verma et al. 2007; Aggarwal \& Keenan 2007, 2008) in electron excitation data for argon-like nickel, initiated by its identification in numerous astrophysical plasmas (consult Verma et al. 2007 for an exhaustive up-to-date bibliography). The most recent studies of the Intra-Cluster Medium, as discussed by Werner et al. (2008), put constraints on supernova models by using $\mathrm{Ni} / \mathrm{Fe}$ abundance patterns in the ejecta of type Ia supernovae. In addition, from the Mass Time-of-Flight Spectrometer data accumulated during the first decade of SOHO's operation, Karrer et al. (2007) inferred charge-state distributions, isotopic composition, and the elemental $\mathrm{Ni} / \mathrm{Fe}$ ratio of the solar wind, and confirmed that both nickel and iron become enriched in the solar corona.

The significance of reliable atomic data has been demonstrated by Churazov et al. (2004) through the modeling and interpretation of the 5-9 keV spectrum from the multi-temperature core of the Perseus galaxy cluster. In their study, Churazov et al. (2004) used APEC (Smith et al. 2001) and MEKAL (Mewe et al. 1985) models, both having the redshift of major line energies, the heavy element abundances, and the plasma temperatures as free parameters. It has been shown that the MEKAL model yields the best description of the spectra only when nickel is overabundant relative to iron by a factor of $\sim 2$ compared to solar. However, this enhancement is not required by the simulations of the APEC code (http: //cxc . harvard.edu/atomdb) that uses updated atomic data.

The present computational study is part of an ongoing investigation of $\mathrm{DR}$ processes in argon-like ions (Nikolić et al. 2007, 2009, 2010), and deals with $\Delta n_{\mathrm{c}}=0,1$ ionic core excitations and associated dielectronic resonances that dominate electron-ion recombination in photoionized plasmas. The theoretical foundation and computational method we use for the DR calculations are found elsewhere (Badnell et al. 2003) and here we only outline the essence. With the use of the open-source AUTOSTRUCTURE code (Badnell 1986, 1997), we carry out MCBP computations of energy levels and decay rates in an intermediate coupling scheme for Ar-like K II, Ca III, Sc IV, Ti V, V VI, Cr VII, Mn VIII, Fe IX, Co X, Ni XI, Cu XII, and Zn XIII ions. In order to account efficiently for all DR contributions coming from numerous Rydberg series of resonances and offer them to the plasma modeling community in convenient level-resolved format, we further enforce the independent-processes, isolatedresonance, distorted-wave (IPIRDW) approximation (Pindzola et al. 1992).

In the remainder of this paper, we will proceed as follows. Section 2 discusses the process of electron-ion recombination and transparently outlines the main relations and equations arising within the adopted methodology. A comparative overview of existing atomic structure in argon-like ions is provided throughout Sect. 3, and an analysis of the results is presented in Sect. 4.

\section{Elementary processes of relevance}

The contribution of the photorecombination process of an ionization state $q+$ from a single partial wave $(J, \pi)$ can be described as

$$
\begin{aligned}
{\left[\mathrm{e}^{-}+\mathrm{I}^{(q)+}\left(3 \mathrm{~s}^{2} 3 \mathrm{p}^{6}\right)\right]_{J, \pi} } & \rightarrow \mathrm{I}^{(q-1)+* *}\left(\begin{array}{c}
3 \mathrm{~s}^{2} 3 \mathrm{p}^{5} 3 \mathrm{~d} n \ell \\
3 \mathrm{~s} 3 \mathrm{p}^{6} 3 \mathrm{~d} n \ell \\
3 \mathrm{~s}^{2} 3 \mathrm{p}^{5} 4 \ell^{\prime} n \ell \\
3 \mathrm{~s} 3 \mathrm{p}^{6} 4 \ell^{\prime} n \ell
\end{array}\right)_{J, \pi} \\
\mathrm{RR} \mathrm{I}^{\mathrm{DR}} \downarrow & \mathrm{I}^{(q-1)+*}\left(3 \mathrm{~s}^{2} 3 \mathrm{p}^{6} n \ell\right)+\omega .
\end{aligned}
$$

Here $c$ is a continuum state consisting of an initial electron incident upon the target ion $\mathrm{I}^{(q)+}$ that is either directly captured, via radiative recombination (RR), to a $\mathrm{I}^{(q-1)+*}$ bound state, $b$, or captured into an autoionizing $\mathrm{I}^{(q-1)+* *}$ doubly-excited state, $d$, that undergoes subsequent radiative decay to the same final bound state, $b$, completing the DR process. The present work investigates $\{3 \mathrm{~s}, 3 \mathrm{p}\} \rightarrow\left\{3 \mathrm{~d}, 4 \ell^{\prime}\right\}$ inelastic excitations from the $\mathrm{I}^{(q)+}$ ground state that give rise to Rydberg series of autoionizing states formed by the capture of the scattered electron. Each Rydberg series of resonances will converge to a corresponding threshold, given in Tables 3-7.

Within the adopted IPIRDW framework, our calculations rely on lowest-order perturbation theory to compute Lorentzian resonance profiles as a function of the electron's center-of-mass (c.m.) energy $\epsilon$. The total DR cross section is then given as

$\sigma^{\mathrm{DR}}(\epsilon)=\frac{1}{\epsilon} \frac{2}{\pi} \sum_{J, \pi} \sum_{b} \sum_{d}^{N^{J, \pi}} \frac{\mathcal{S}_{c \rightarrow b}^{d} / \bar{\Gamma}_{d}}{1+\varepsilon_{d}^{2}}$

Here $\varepsilon_{d}=2\left(\epsilon-\epsilon_{d}\right) / \bar{\Gamma}_{d}$ is the reduced c.m. energy at which the (partial) integrated resonant strength is

$$
\mathcal{S}_{c \rightarrow b}^{d}\left[\mathrm{Mb} \mathrm{Ry}^{2}\right]=2.674 \times 10^{-14} \frac{g_{d}}{2 g_{\text {ion }}} \frac{A_{d \rightarrow c}^{a} A_{d \rightarrow b}^{r}}{\sum_{c^{\prime}} A_{d \rightarrow c^{\prime}}^{a}+\sum_{s} A_{d \rightarrow s}^{r}},
$$

with $g_{d}$ and $g_{\text {ion }}$ as statistical weights of the resonant state $d$ in the recombined ion and the ground state of the target ion, respectively. The summations over $c^{\prime}$ and $s$ in Eq. (3) cover all states that are attainable from resonant state $d$ either by radiative decay or by autoionization, with corresponding rates given in inverse seconds. Hence, the sum over $s$ includes not only bound states that are below the first ionization limit of the recombined ion, $E_{\text {th }}^{(1)}$, but also may consider a radiative cascade through other autoionizing states resulting in the total radiative rate, $A_{d}^{r}$. The sum over $c^{\prime}$ accounts for resonant scattering (excitation) and amounts to the total autoionization rate, $A_{d}^{a}$. In Eqs. (2)-(3) we implicitly assume that both the resonance position, $\epsilon_{d}=E_{d}-E_{\text {th }}^{(1)}$, and the total width, $\bar{\Gamma}_{d}=\hbar\left(A_{d}^{a}+A_{d}^{r}\right)$, are in Rydberg units. In addition, the summation over $d$ spans all of the $N^{J, \pi}$ doubly excited states of given parity $\pi$ and total angular momentum $J$ that are formed through Eq. (1) for $3 \leq n \leq 1000$ and $0 \leq \ell \leq 10$, wherein the index $b$ considers all accessible bound states.

The DR/RR rate coefficient (in units of $\mathrm{cm}^{3} \mathrm{~s}^{-1}$ ) for $\mathrm{I}^{q+}$ ions in a plasma with a Maxwellian electron energy distribution $f_{\mathrm{MB}}(\epsilon, T)$ is given by

$\alpha^{\mathrm{DR} / \mathrm{RR}}(T)=\int_{0}^{\infty} v(\epsilon) \sigma^{\mathrm{DR} / \mathrm{RR}}(\epsilon) f_{\mathrm{MB}}(\epsilon, T) \mathrm{d} \epsilon$ 
D. Nikolić et al.: Dielectronic recombination of argon-like ions

Table 1. Radial scaling parameters, $\lambda_{n l}$, for the $n=3$ valence orbitals optimized in the Slater-type-orbital model potential.

\begin{tabular}{lllllllllllll}
\hline \hline & $\mathrm{K}^{+}$ & $\mathrm{Ca}^{2+}$ & $\mathrm{Sc}^{3+, a}$ & $\mathrm{Ti}^{4+, b}$ & $\mathrm{~V}^{5+}$ & $\mathrm{Cr}^{6+}$ & $\mathrm{Mn}^{7+}$ & $\mathrm{Fe}^{8+}$ & $\mathrm{Co}^{9+}$ & $\mathrm{Ni}^{10+}$ & $\mathrm{Cu}^{11+}$ & $\mathrm{Zn}^{12+}$ \\
\hline$\lambda_{3 s}$ & 1.09548 & 1.09548 & 1.08366 & 1.05653 & 1.07085 & 1.07085 & 1.07085 & 1.05990 & 1.0000 & 1.0000 & 1.0000 & 1.0000 \\
$\lambda_{3 p}$ & 1.11185 & 1.10545 & 0.97900 & 0.98950 & 1.14255 & 1.11729 & 1.09171 & 1.07936 & 1.0693 & 1.0608 & 1.0453 & 1.0318 \\
$\lambda_{3 d}$ & 1.13830 & 1.16918 & 1.27700 & 1.17400 & 0.89575 & 0.87575 & 0.87575 & 0.84905 & 0.8391 & 0.8191 & 0.8191 & 0.8191 \\
\hline
\end{tabular}

Notes. Details about the full optimization procedure are reported earlier by Nikolić et al. (2009). adopted from: ${ }^{(a)}$ Nikolić et al. (2010); ${ }^{(b)}$ Nikolić et al. (2009).

Table 2. Dominant radiative transition data from the ground state of the recombining ion $3 p^{6}\left({ }^{1} S_{0}\right) \rightarrow 3 p^{5} 3 d\left({ }^{1} P_{1}^{\mathrm{o}}\right)$, where $v_{(u)}^{[ \pm p]}$ denotes $v(u) \times 10^{ \pm p}$.

\begin{tabular}{|c|c|c|c|c|c|c|c|c|c|}
\hline Ion & $\begin{array}{c}A^{r} \\
\left(\mathrm{~ns}^{-1}\right)\end{array}$ & $\begin{array}{c}S \\
\text { (a.u.) }\end{array}$ & $f$ & Ref. & Ion & $\begin{array}{c}A^{r} \\
\left(\mathrm{~ns}^{-1}\right) \\
\end{array}$ & $\begin{array}{c}S \\
\text { (a.u.) } \\
\end{array}$ & $f$ & Ref. \\
\hline \multirow{3}{*}{$\mathrm{K}^{+}$} & $0.2785^{[+2]}$ & 5.004 & 3.070 & Present $^{a}$ & \multirow{5}{*}{$\mathrm{Ca}^{2+}$} & $0.836^{[+2]}$ & 5.678 & 4.818 & Present $^{a}$ \\
\hline & $0.2224^{[+2]}$ & 4.147 & 2.513 & $\mathrm{MCHF}^{\mathrm{c}}$ & & $0.83_{(15)}^{[+2]}$ & 5.64 & $4.75_{(88)}$ & $\mathrm{NIST}^{\mathrm{b}, \S}$ \\
\hline & $1.4610^{[+2]}$ & 2.448 & 3.313 & Present $^{a}$ & & $0.638^{[+2]}$ & 4.614 & 3.834 & $\mathrm{MCHF}^{\mathrm{c}}$ \\
\hline \multirow[t]{3}{*}{$\mathrm{V}^{5+}$} & $1.46^{[+2]}$ & 2.45 & 3.31 & $\mathrm{NIST}^{\mathrm{b} \dagger}$ & & $0.682^{[+2]}$ & 4.210 & 3.688 & $\mathrm{CIV}^{\mathrm{j}}$ \\
\hline & $1.8447^{[+2]}$ & 2.968 & 4.070 & $\mathrm{MCHF}^{c}$ & & $1.65^{[+2]}$ & 2.04 & 3.05 & Present $^{a}$ \\
\hline & $1.858^{[+2]}$ & 1.754 & 2.874 & Present $^{a}$ & \multirow[t]{5}{*}{$\mathrm{Cr}^{6+}$} & $1.67^{[+2]}$ & 2.06 & 3.09 & $\mathrm{NIST}^{b}{ }^{\S}$ \\
\hline \multirow[t]{3}{*}{$\mathrm{Mn}^{7+}$} & $1.85^{[+2]}$ & 1.75 & 2.86 & $\mathrm{NIST}^{b}, \S$ & & $2.05^{[+2]}$ & 2.43 & 3.69 & $\mathrm{MCHF}^{c}$ \\
\hline & $1.963^{[+2]}$ & 2.033 & 3.229 & $\mathrm{MCHF}^{c}$ & & $2.009^{[+2]}$ & 1.489 & 2.645 & Present $^{a}$ \\
\hline & $2.190^{\lfloor+2]}$ & 1.300 & 2.486 & Present $^{a}$ & & $2.01^{[+2]}$ & 1.49 & 2.65 & $\mathrm{NIST}^{b}, \S$ \\
\hline \multirow{5}{*}{$\mathrm{Co}^{9+}$} & $2.200^{[+2]}$ & 1.300 & 2.500 & $\mathrm{NIST}^{b \ddagger}$ & & $2.097^{[+2]}$ & 1.724 & 2.958 & $\mathrm{MCHF}^{c}$ \\
\hline & $2.224^{[+2]}$ & 1.482 & 2.726 & $\mathrm{MCHF}^{c}$ & \multirow{6}{*}{$\mathrm{Fe}^{8+}$} & $2.438^{[+2]}$ & 1.678 & 3.054 & $\mathrm{FAC}^{d}{ }^{d}$ \\
\hline & $1.448^{[+2]}$ & 1.409 & 2.628 & $\mathrm{TDCHF}^{f}$ & & $2.266^{[+2]}$ & 1.680 & 2.983 & $\mathrm{CIV}^{e}$ \\
\hline & $2.339^{[+2]}$ & 1.132 & 2.317 & Present $^{a}$ & & $1.356^{[+2]}$ & 1.073 & 2.843 & $\mathrm{TDCHF}^{f}$ \\
\hline & $2.340^{[+2]}$ & 1.130 & 2.320 & NIST $^{b \dagger}$ & & $2.889^{[+2]}$ & & & SELT $^{g}$ \\
\hline \multirow{5}{*}{$\mathrm{Ni}^{10+}$} & $2.345^{[+2]}$ & 1.288 & 2.528 & $\mathrm{MCHF}^{c}$ & & $2.404^{[+2]}$ & 1.637 & 2.99 & IRON $^{h}$ \\
\hline & $1.537^{[+2]}$ & 0.808 & 2.444 & $\mathrm{TDCHF}^{f}$ & & $2.530^{[+2]}$ & 1.01 & 2.204 & Present $^{a}$ \\
\hline & $2.786^{[+2]}$ & 1.274 & 2.657 & $\mathrm{FAC}^{h}$ & \multirow{6}{*}{$\mathrm{Cu}^{11+}$} & $2.501_{(30)}^{[+2]}$ & $1.07_{(11)}$ & $2.29_{(7)}$ & $\mathrm{NIST}^{b}, \S$ \\
\hline & $2.841^{[+2]}$ & 1.204 & 2.575 & $\mathrm{CIV}^{i}{ }^{i}$ & & $2.462^{[+2]}$ & 1.13 & 2.355 & $\mathrm{MCHF}^{c}$ \\
\hline & $2.720^{\lfloor+2]}$ & 0.906 & 2.101 & Present $^{a}$ & & $1.624^{[+2]}$ & 1.08 & 2.285 & $\mathrm{TDCHF}^{f}$ \\
\hline \multirow{3}{*}{$\mathrm{Zn}^{12+}$} & $2.653_{(33)}^{[+2]}$ & $0.99_{(11)}$ & $2.21_{(7)}$ & $\mathrm{NIST}^{b}, \S$ & & & & & \\
\hline & $2.576^{[+2]}$ & 1.001 & 2.204 & $\mathrm{MCHF}^{c}$ & & & & & \\
\hline & $1.708^{[+2]}$ & 0.958 & 2.145 & $\mathrm{TDCHF}^{f}$ & & & & & \\
\hline
\end{tabular}

Notes. In the case of $\mathrm{Sc}^{3+}$ and $\mathrm{Ti}^{4+}$ ions, refer to Nikolić et al. (2010) and Nikolić et al. (2009), respectively.

References. Assigned uncertainties: ${ }^{(\dagger)}<18 \%$, $\left.{ }^{(}\right)<25 \%$; ${ }^{(a)}$ present work: 2894-level MCBP results; ${ }^{(b)}$ critically compiled experimental data of Shirai et al. (2000); ${ }^{(c)}$ MCHF results of Froese-Fischer \& Tachiev (2006); ${ }^{(d)}$ 6284-level FAC results of Aggarwal et al. (2006); ${ }^{(e)}$ CIV3 results of Verma et al. (2006); ${ }^{(f)}$ single-config. TDCHF results of Ghosh \& Mukherjee (1997); ${ }^{(g)}$ semiempirical results of Loginov \& Tuchkin (2002); ${ }^{(h)}$ 6164-level FAC results of Aggarwal \& Keenan (2008); ${ }^{(i)}$ restricted CIV3 results of Verma et al. (2007); ${ }^{(j)}$ 18-config. CIV3 LS results of Baluja (1986); $\left.{ }^{(}\right)$extrapolated along the isoelectronic sequence.

and conveniently modeled using physically-motivated fitting formulae (Burgess 1965)

$\alpha^{\mathrm{DR}}(T)=T^{-3 / 2} \sum_{i} c_{i} \exp \left(-E_{i} / T\right)$ and

$\alpha^{\mathrm{RR}}(T)=A\left[\sqrt{T / T_{0}}\left(1+\sqrt{T / T_{0}}\right)^{p_{-}}\left(1+\sqrt{T / T_{1}}\right)^{p_{+}}\right]^{-1}$,

where the more general form $p_{ \pm}=1 \pm B \pm C \exp \left(-T_{2} / T\right)$ of Verner \& Ferland (1996) is used here. The fitted DR rate coefficients given by Eq. (5) are relatively smooth and broad curves with several local maxima, each at a particular temperature $T_{i}=2 E_{i} / 3$, with $E_{i}$ given in Table 8 . On the other hand, the RR rate coefficients given by Eq. (6) decrease monotonically with temperature; the fitting coefficients $A, B, C$, and $T_{0,1,2}$ are given in Table 9. These nonlinear least-squares fits are accurate to better than $1 \%$ over $\left(10^{1}-10^{7}\right) q^{2} \mathrm{~K}$ with the correct asymptotic forms outside of this temperature range.

\section{Atomic structure}

Before performing the electron-ion scattering calculation in Eq. (1), it is necessary to obtain an accurate description of the Ar-like target-ion states. Our approach for the entire Ar-like isoelectronic sequence is essentially the same as what was done earlier for Ti V (Nikolić et al. 2009). Since the dominant DR contribution is due to the $e^{-}+3 \mathrm{~s}^{2} 3 \mathrm{p}^{6} \rightarrow 3 \mathrm{~s}^{2} 3 \mathrm{p}^{5} 3 \mathrm{~d} n \ell$ capture, we are particularly concerned with obtaining highly-accurate wavefunctions for both the $3 \mathrm{~s}^{2} 3 \mathrm{p}^{6}$ ground state and the $3 \mathrm{~s}^{2} 3 \mathrm{p}^{5} 3 \mathrm{~d}$ excited state. However, we also need to consider the $3 s^{3} 3 p^{6} 3 d$, $3 s^{2} 3 p^{5} 4 \ell^{\prime}$, and $3 s 3 p^{6} 4 \ell^{\prime}$ target states that also contribute to the DR rate coefficient via Eq. (1). Single and double promotions out of these configurations are also included in our total configuration expansion for each target state.

With this configuration basis, the $1 \mathrm{~s}, 2 \mathrm{~s}, 2 \mathrm{p}, 3 \mathrm{~s}, 3 \mathrm{p}$, and $3 \mathrm{~d}$ Slater-type orbitals were determined by varying the radial scaling parameters, $\lambda_{n \ell}$, to minimize the equally-weighted-sum of MCBP eigenenergies of the seventeen lowest states, using the 
A\&A 516, A97 (2010)

Table 3. The lowest $\Delta n_{\mathrm{c}}=0,1$ core excitation thresholds (in Rydbergs) for K II and Ca III.

\begin{tabular}{|c|c|c|c|c|c|c|c|c|c|c|c|}
\hline \multicolumn{8}{|c|}{$\mathrm{K}^{+}$} & \multicolumn{4}{|c|}{$\mathrm{Ca}^{2+}$} \\
\hline K & Config. & Level(mix) & Present $^{a}$ & $\mathrm{NIST}^{b}$ & $\mathrm{MCHF}^{c}$ & $\mathrm{~K}$ & Config. & Level(mix) & Present $^{a}$ & $\mathrm{NIST}^{d}$ & $\mathrm{MCHF}^{c}$ \\
\hline 1 & $3 s^{2} 3 p^{6}$ & ${ }^{1} \mathrm{~S}_{0}(96.3 \%)$ & 0.000000 & 0.000000 & 0.000000 & 1 & $3 s^{2} 3 p^{6}$ & ${ }^{1} \mathrm{~S}_{0}(99.0 \%)$ & 0.000000 & 0.000000 & 0.000000 \\
\hline 2 & $3 s^{2} 3 p^{5} 4 s$ & ${ }^{3} \mathrm{P}_{2}^{\mathrm{o}}(93.2 \%)$ & 1.480381 & 1.480834 & 1.400644 & 2 & $3 s^{2} 3 p^{5} 3 d$ & ${ }^{3} \mathrm{P}_{0}^{\mathrm{o}}(95.7 \%)$ & 1.879530 & 1.853273 & 1.760169 \\
\hline 3 & $3 s^{2} 3 p^{5} 4 s$ & ${ }^{3} \mathrm{P}_{1}^{2}(59.7 \%)$ & 1.487826 & 1.487481 & 1.408491 & 3 & $3 s^{2} 3 p^{5} 3 d$ & ${ }^{3} \mathrm{P}_{1}^{\mathrm{o}}(95.6 \%)$ & 1.884279 & 1.857636 & 1.764514 \\
\hline 4 & $3 s^{2} 3 p^{5} 3 d$ & ${ }^{3} \mathrm{P}_{0}^{\mathrm{O}}(70.9 \%)$ & 1.490116 & 1.489303 & 1.431384 & 4 & $3 s^{2} 3 p^{5} 3 d$ & ${ }^{3} \mathrm{P}_{2}^{\mathrm{o}}(95.2 \%)$ & 1.893907 & 1.866664 & 1.773134 \\
\hline 5 & $3 s^{2} 3 p^{5} 3 d$ & ${ }^{3} \mathrm{P}_{1}^{\mathrm{o}}(59.5 \%)$ & 1.499706 & 1.498961 & 1.430119 & 5 & $3 s^{2} 3 p^{5} 3 d$ & ${ }^{3} \mathrm{~F}_{4}^{\mathrm{O}}(95.1 \%)$ & 1.952880 & 1.934711 & 1.844004 \\
\hline 6 & $3 s^{2} 3 p^{5} 3 d$ & ${ }^{3} \mathrm{P}_{2}^{\mathrm{o}}(81.1 \%)$ & 1.505571 & 1.502934 & 1.436726 & 6 & $3 s^{2} 3 p^{5} 3 d$ & ${ }^{3} \mathrm{~F}_{3}^{\mathrm{o}}(93.9 \%)$ & 1.963210 & 1.944456 & 1.853118 \\
\hline 7 & $3 s^{2} 3 p^{5} 4 s$ & ${ }^{3} \mathrm{P}_{0}^{\mathrm{o}}(82.4 \%)$ & 1.506735 & 1.504916 & 1.414847 & 7 & $3 s^{2} 3 p^{5} 3 d$ & ${ }^{3} \mathrm{~F}_{2}^{\mathrm{o}}(94.0 \%)$ & 1.972601 & 1.953156 & 1.861216 \\
\hline 8 & $3 s^{2} 3 p^{5} 4 s$ & ${ }^{1} \mathrm{P}_{1}^{\mathrm{o}}(74.8 \%)$ & 1.517409 & 1.516872 & 1.437439 & 8 & $3 s^{2} 3 p^{5} 3 d$ & ${ }^{1} \mathrm{D}_{2}^{\mathrm{o}}(58.9 \%)$ & 2.086507 & 2.057880 & 1.972081 \\
\hline 9 & $3 s^{2} 3 p^{5} 3 d$ & ${ }^{3} \mathrm{~F}_{4}^{1}(82.4 \%)$ & 1.547896 & 1.549602 & 1.480702 & 9 & $3 s^{2} 3 p^{5} 3 d$ & ${ }^{3} \mathrm{D}_{3}^{\mathrm{o}}(53.7 \%)$ & 2.080602 & 2.062503 & 1.976286 \\
\hline 10 & $3 s^{2} 3 p^{5} 3 d$ & ${ }^{3} \mathrm{~F}_{3}^{\mathrm{o}}(81.1 \%)$ & 1.555741 & 1.556728 & 1.488382 & 10 & $3 s^{2} 3 p^{5} 3 d$ & ${ }^{3} \mathrm{D}_{1}^{\mathrm{o}}(92.9 \%)$ & 2.093231 & 2.072514 & 1.985320 \\
\hline 11 & $3 s^{2} 3 p^{5} 3 d$ & ${ }^{3} \mathrm{~F}_{2}^{\mathrm{o}}(81.3 \%)$ & 1.562998 & 1.563029 & 1.494070 & 11 & $3 s^{2} 3 p^{5} 3 d$ & ${ }^{3} \mathrm{D}_{2}^{\mathrm{o}}(54.0 \%)$ & 2.095232 & 2.072117 & 1.984866 \\
\hline 12 & $3 s^{2} 3 p^{5} 3 d$ & ${ }^{3} \mathrm{D}_{3}^{\mathrm{o}}(35.7 \%)$ & 1.633040 & 1.634775 & 1.561671 & 12 & $3 s^{2} 3 p^{5} 3 d$ & ${ }^{1} \mathrm{~F}_{3}^{\mathrm{o}}(57.8 \%)$ & 2.081461 & 2.097941 & 1.995780 \\
\hline 13 & $3 s^{2} 3 p^{5} 3 d$ & ${ }^{1} \mathrm{D}_{2}^{\mathrm{o}}(41.6 \%)$ & 1.639503 & 1.634350 & 1.560836 & 13 & $3 s^{2} 3 p^{5} 4 s$ & ${ }^{3} \mathrm{P}_{2}^{\mathrm{o}}(99.4 \%)$ & 2.313063 & 2.210253 & 2.114045 \\
\hline 14 & $3 s^{2} 3 p^{5} 3 d$ & ${ }^{3} \mathrm{D}_{1}^{\mathrm{o}}(71.5 \%)$ & 1.644348 & 1.644371 & 1.570061 & 14 & $3 s^{2} 3 p^{5} 4 s$ & ${ }^{3} \mathrm{P}_{1}^{\mathrm{o}}(88.0 \%)$ & 2.327118 & 2.222858 & 2.126335 \\
\hline 15 & $3 s^{2} 3 p^{5} 3 d$ & ${ }^{3} \mathrm{D}_{2}^{\mathrm{o}}(41.9 \%)$ & 1.645874 & 1.643859 & 1.569134 & 15 & $3 s^{2} 3 p^{5} 4 s$ & ${ }^{3} \mathrm{P}_{0}^{0}(99.4 \%)$ & 2.341390 & 2.238180 & 2.140287 \\
\hline 16 & $3 s^{2} 3 p^{5} 3 d$ & ${ }^{1} \mathrm{~F}_{3}^{\mathrm{o}}(34.1 \%)$ & 1.645999 & 1.645919 & 1.571404 & 16 & $3 s^{2} 3 p^{5} 4 s$ & ${ }^{1} \mathrm{P}_{1}^{\mathrm{o}}(87.6 \%)$ & 2.369020 & 2.257176 & 2.160581 \\
\hline 17 & $3 s^{2} 3 p^{5} 4 p$ & ${ }^{3} \mathrm{~S}_{1}(97.7 \%)$ & 1.663215 & 1.669479 & 1.591351 & 17 & $3 s^{2} 3 p^{5} 3 d$ & ${ }^{1} \mathrm{P}_{1}^{\mathrm{o}}(63.1 \%)^{\star}$ & 2.545658 & 2.545658 & 2.492690 \\
\hline 18 & $3 s^{2} 3 p^{5} 4 p$ & ${ }^{3} \mathrm{D}_{3}(99.9 \%)$ & 1.693675 & 1.698454 & 1.618318 & & & & & & \\
\hline 19 & $3 s^{2} 3 p^{5} 4 p$ & ${ }^{3} \mathrm{D}_{2}(75.5 \%)$ & 1.697258 & 1.701166 & 1.621064 & & & & & & \\
\hline 20 & $3 s^{2} 3 p^{5} 4 p$ & ${ }^{3} \mathrm{D}_{1}(76.3 \%)$ & 1.705934 & 1.708872 & 1.628229 & & & & & & \\
\hline 21 & $3 s^{2} 3 p^{5} 4 p$ & ${ }^{1} \mathrm{D}_{2}(51.0 \%)$ & 1.712827 & 1.714552 & 1.633790 & & & & & & \\
\hline 22 & $3 s^{2} 3 p^{5} 4 p$ & ${ }^{1} \mathrm{P}_{1}(52.5 \%)$ & 1.724516 & 1.724477 & 1.643195 & & & & & & \\
\hline 23 & $3 s^{2} 3 p^{5} 4 p$ & ${ }^{3} \mathrm{P}_{2}(55.9 \%)$ & 1.727938 & 1.728285 & 1.646621 & & & & & & \\
\hline 24 & $3 s^{2} 3 p^{5} 4 p$ & ${ }^{3} \mathrm{P}_{0}(99.5 \%)$ & 1.730260 & 1.729293 & 1.648054 & & & & & & \\
\hline 25 & $3 s^{2} 3 p^{5} 4 p$ & ${ }^{3} \mathrm{P}_{1}(65.5 \%)$ & 1.733330 & 1.732598 & 1.650821 & & & & & & \\
\hline 26 & $3 s^{2} 3 p^{5} 4 p$ & ${ }^{1} \mathrm{~S}_{0}(95.2 \%)$ & 1.829238 & 1.774895 & 1.702454 & & & & & & \\
\hline \multirow[t]{2}{*}{27} & $3 s^{2} 3 p^{5} 3 d$ & ${ }^{1} \mathrm{P}_{1}^{\mathrm{o}}(90.5 \%)^{\star}$ & 1.840715 & 1.840373 & 1.817999 & & & & & & \\
\hline & $3 s^{2} 3 p^{5} 4 d$ & ${ }^{1} \mathrm{P}_{1}^{\mathrm{l}}(90.0 \%)$ & 2.033261 & 2.033256 & & & & & & & \\
\hline
\end{tabular}

Notes. For brevity, we show only the core excitations that are below the dominant $3 \mathrm{p}^{6}\left({ }^{1} \mathrm{~S}_{0}\right) \rightarrow 3 \mathrm{p}^{5} 3 \mathrm{~d}\left({ }^{1} \mathrm{P}_{1}^{\mathrm{o}}\right)$ threshold. The exception is the $3 \mathrm{p}^{5} 4 \mathrm{~d}\left({ }^{1} \mathrm{P}_{1}^{\mathrm{o}}\right)$ threshold in K II whose configuration is marked as questionable by Sugar \& Corliss (1985) and only recently properly identified by Pettersen et al. (2007). ${ }^{(a)}$ present work: 51-level $\left(\mathrm{K}^{+}\right)$and 69 -level $\left(\mathrm{Ca}^{2+}\right)$ MCBP results; ${ }^{(b)} \mathrm{UV}$ spark spectroscopy experimental data of Pettersen et al. (2007); ${ }^{(c)}$ MCHF results of Froese-Fischer \& Tachiev (2006); ${ }^{(d)}$ critically compiled experimental data of Sugar \& Corliss (1985); $(\star)$ dominant excitation threshold - see Table 2.

model potential of Burgess et al. (1989) with mass-velocity and Darwin corrections. The values of the radial scaling parameters for closed-core orbitals $(1 \mathrm{~s}, 2 \mathrm{~s}$, and $2 \mathrm{p})$ remained throughout the optimization in near proximity to their default value of 1.0 . We then applied small adjustments to the $3 \ell$ scaling parameters in order to reproduce the NIST experimental $3 \mathrm{p} \rightarrow 3 \mathrm{~d}$ radiative data of Shirai et al. (2000). The resultant $\lambda_{n \ell}$ values are listed in Table 1 and the radiative data are listed in Table 2. As is seen in Table 2, our target description is such that the present oscillator strengths are in excellent agreement with the experimental values.

We have also confirmed that the computed eigenenergies were stationary with respect to the small variations in $\lambda_{n \ell}$ values that were used to fine-tune the $3 p \rightarrow 3 d$ oscillator strengths. Indeed, as is seen in Tables 3, 4, 5, 6, and 7 for the lowest-lying Ar-like states, our computed energies are in excellent agreement with the experimental values given in the NIST Atomic Spectra Database (Sugar \& Corliss 1985; Shirai et al. 2000; Pettersen et al. 2007). In the case of higher ionization stages, the atomic data produced by Froese-Fischer \& Tachiev (2006) using the multi-configurational Hartree-Fock (MCHF) method, or by a non-relativistic single-configuration approach of Ghosh \& Mukherjee (1997), are up to 7.2\% lower than the NIST values. In contrast to the present study, the calculations of Froese-Fischer $\&$ Tachiev (2006) lacked the important $3 \mathrm{p}^{2} \rightarrow 3 \mathrm{~d}^{2}$ excitation contributions, which is essential for improving the radiative transition data. Furthermore, for higher degrees of ionization, only the terms of the $3 s^{2} 3 p^{5} 3 d$ configuration were retained in their calculations. The basic CIV3 atomic data for Fe IX produced by Verma et al. (2006) are noticeably closer to the present results than those of Aggarwal et al. (2006) using the fully relativistic multi-configurational Flexible Atomic Code (FAC) of $\mathrm{Gu}$ (2004).

\section{DR results}

To treat the DR processes occurring in Eq. (1), all possible continuum and resonance wavefunctions are constructed by coupling an appropriate distorted-wave free $\epsilon \ell$ or bound $n \ell$ orbital to each target configuration wavefunction, as obtained in the previous section. The energies, radiative rates, and Auger rates of each resonance are then computed and used in Eqs. (2) and (4) to produce Maxwellian rate coefficients that are shown in Figs. 7-9. In all the figures, the bars indicating the collisionally ionized zone were obtained assuming the conditions of coronal equilibrium with electron-collisional plasma temperatures for which the fractional abundance of the target ion in question surpasses $10 \%$ of its peak value (Bryans et al. 2008). Similar approximate temperature indicators for gas in photoionization equilibrium have been computed using Cloudy 
Table 4. The lowest $\Delta n_{\mathrm{c}}=0$ core excitation thresholds (in Rydbergs) for V VI and Cr VII.

\begin{tabular}{|c|c|c|c|c|c|c|c|c|c|}
\hline \multirow[b]{2}{*}{ K } & \multirow[b]{2}{*}{ Config. } & \multicolumn{4}{|c|}{$\mathrm{V}^{5+}$} & \multicolumn{4}{|c|}{$\mathrm{Cr}^{6+}$} \\
\hline & & Level(mix) & Present $^{a}$ & $\mathrm{NIST}^{b}$ & $\mathrm{MCHF}^{c}$ & Level(mix) & Present $^{a}$ & $\mathrm{NIST}^{b}$ & $\mathrm{MCHF}^{c}$ \\
\hline 1 & $3 s^{2} 3 p^{6}$ & ${ }^{\mathrm{T}} \mathrm{S}_{0}(96.4 \%)$ & 0.000000 & 0.000000 & 0.000000 & ${ }^{1} \mathrm{~S}_{0}(96.6 \%)$ & 0.000000 & 0.000000 & 0.000000 \\
\hline 2 & $3 s^{2} 3 p^{5} 3 d$ & ${ }^{3} \mathrm{P}_{0}^{\mathrm{o}}(97.0 \%)$ & 2.739060 & 2.808068 & 2.806866 & ${ }^{3} \mathrm{P}_{0}^{\mathrm{o}}(97.1 \%)$ & 3.059906 & 3.109055 & 3.110232 \\
\hline 3 & $3 s^{2} 3 p^{5} 3 d$ & ${ }^{3} \mathrm{P}_{1}^{\mathrm{o}}(96.9 \%)$ & 2.748293 & 2.819413 & 2.818090 & ${ }^{3} \mathrm{P}_{1}^{0}(96.9 \%)$ & 3.072680 & 3.123582 & 3.124881 \\
\hline 4 & $3 s^{2} 3 p^{5} 3 d$ & ${ }^{3} \mathrm{P}_{2}^{\mathrm{o}}(96.7 \%)$ & 2.767020 & 2.842952 & 2.841350 & ${ }^{3} \mathrm{P}_{2}^{1}(96.6 \%)$ & 3.098672 & 3.154233 & 3.155270 \\
\hline 5 & $3 s^{2} 3 p^{5} 3 d$ & ${ }^{3} \mathrm{~F}_{4}^{\mathrm{o}}(97.3 \%)$ & 2.896923 & 2.941329 & 2.944497 & ${ }^{3} \mathrm{~F}_{4}^{\mathrm{O}}(97.4 \%)$ & 3.234927 & 3.258178 & 3.264649 \\
\hline 6 & $3 s^{2} 3 p^{5} 3 d$ & ${ }^{3} \mathrm{~F}_{3}^{\mathrm{o}}(96.2 \%)$ & 2.906694 & 2.961235 & 2.963277 & ${ }^{3} \mathrm{~F}_{3}^{\mathrm{o}}(95.7 \%)$ & 3.247762 & 3.282128 & 3.287197 \\
\hline 7 & $3 s^{2} 3 p^{5} 3 d$ & ${ }^{3} \mathrm{~F}_{2}^{\mathrm{O}}(96.6 \%)$ & 2.917192 & 2.981802 & 2.982698 & ${ }^{3} \mathrm{~F}_{2}^{\mathrm{o}}(96.2 \%)$ & 3.262960 & 3.308454 & 3.312214 \\
\hline 8 & $3 s^{2} 3 p^{5} 3 d$ & ${ }^{1} \mathrm{D}_{2}^{\mathrm{o}}(71.5 \%)$ & 3.110770 & 3.145142 & 3.152963 & ${ }^{1} \mathrm{D}_{2}^{\mathrm{o}}(67.4 \%)$ & 3.470531 & 3.487258 & 3.497713 \\
\hline 9 & $3 s^{2} 3 p^{5} 3 d$ & ${ }^{3} \mathrm{D}_{3}^{\mathrm{o}}(78.2 \%)$ & 3.119456 & 3.148578 & 3.156780 & ${ }^{3} \mathrm{D}_{3}^{\mathrm{o}}(72.3 \%)$ & 3.478260 & 3.487760 & 3.499169 \\
\hline 10 & $3 s^{2} 3 p^{5} 3 d$ & ${ }^{3} \mathrm{D}_{1}^{\mathrm{o}}(96.8 \%)$ & 3.126389 & 3.170297 & 3.176499 & ${ }^{3} \mathrm{D}_{1}^{\mathrm{o}}(96.8 \%)$ & 3.489568 & 3.515926 & 3.525054 \\
\hline 11 & $3 s^{2} 3 p^{5} 3 d$ & ${ }^{3} \mathrm{D}_{2}^{\mathrm{o}}(71.6 \%)$ & 3.132258 & 3.174174 & 3.180891 & ${ }^{3} \mathrm{D}_{2}^{\mathrm{o}}(71.8 \%)$ & 3.499112 & 3.523110 & 3.532855 \\
\hline 12 & $3 s^{2} 3 p^{5} 3 d$ & ${ }^{1} \mathrm{~F}_{3}^{\mathrm{o}}(77.6 \%)$ & 3.145710 & 3.195308 & 3.203371 & ${ }^{1} \mathrm{~F}_{3}^{\mathrm{o}}(71.3 \%)$ & 3.512852 & 3.546890 & 3.557753 \\
\hline 13 & $3 s^{2} 3 p^{5} 3 d$ & ${ }^{1} \mathrm{P}_{1}^{\mathrm{o}}(95.4 \%)^{\star}$ & 4.059132 & 4.059108 & 4.114305 & ${ }^{1} \mathrm{P}_{1}^{\mathrm{o}}(95.6 \%)^{\star}$ & 4.492846 & 4.492869 & 4.553079 \\
\hline 14 & $3 \mathrm{~s} 3 \mathrm{p}^{6} 3 \mathrm{~d}$ & ${ }^{3} \mathrm{D}_{1}(69.1 \%)$ & 5.013754 & 5.007759 & 5.122273 & ${ }^{3} \mathrm{D}_{1}(71.9 \%)$ & 5.547059 & 5.546697 & 5.667028 \\
\hline 15 & $3 s 3 p^{6} 3 d$ & ${ }^{3} \mathrm{D}_{2}(69.1 \%)$ & 5.017989 & 5.010726 & 5.124985 & ${ }^{3} \mathrm{D}_{2}(71.8 \%)$ & 5.552838 & 5.550917 & 5.670942 \\
\hline 16 & $3 s^{3} p^{6} 3 d$ & ${ }^{3} \mathrm{D}_{3}(69.2 \%)$ & 5.024692 & 5.015474 & 5.129131 & ${ }^{3} \mathrm{D}_{3}(72.0 \%)$ & 5.562141 & 5.557707 & 5.676969 \\
\hline 17 & $3 s 3 p^{6} 3 d$ & ${ }^{1} \mathrm{D}_{2}(63.6 \%)$ & 5.191350 & 5.161717 & 5.318685 & ${ }^{1} \mathrm{D}_{2}(66.4 \%)$ & 5.742414 & 5.721178 & 5.883764 \\
\hline
\end{tabular}

Notes. ${ }^{(a)}$ present work: 2894-level MCBP results; ${ }^{(b)}$ critically compiled experimental data of Shirai et al. (2000); ${ }^{(c)}$ MCHF results of FroeseFischer \& Tachiev (2006); $\left.{ }^{\star}{ }^{\star}\right)$ dominant excitation threshold - see Table 2.

Table 5. The lowest $\Delta n_{\mathrm{c}}=0$ core excitation thresholds (in Rydbergs) for Mn VIII and Fe IX.

\begin{tabular}{|c|c|c|c|c|c|c|c|c|c|c|c|c|}
\hline \multirow[b]{2}{*}{$\mathrm{K}$} & \multirow[b]{2}{*}{ Config. } & \multicolumn{4}{|c|}{$\mathrm{Mn}^{7+}$} & \multicolumn{7}{|c|}{$\mathrm{Fe}^{8+}$} \\
\hline & & Level(mix) & Present $^{a}$ & $\mathrm{NIST}^{b}$ & $\mathrm{MCHF}^{c}$ & Level(mix) & Present $^{a}$ & $\mathrm{NIST}^{b}$ & $\mathrm{MCHF}^{c}$ & $\mathrm{CIV}^{e}$ & IRON $^{f}$ & $\mathrm{FAC}^{g}$ \\
\hline 1 & $3 s^{2} 3 p^{6}$ & ${ }^{1} \mathrm{~S}_{0}(96.7 \%)$ & 0.00000 & 0.00000 & 0.00000 & ${ }^{1} \mathrm{~S}_{0}(96.9 \%)$ & 0.00000 & 0.00000 & 0.00000 & 0.00000 & 0.00000 & 0.0000 \\
\hline $\begin{array}{l}2 \\
3 \\
4\end{array}$ & $\begin{array}{l}3 s^{2} 3 p^{5} 3 d \\
3 s^{2} 3 p^{5} 3 d \\
3 s^{2} 3 p^{5} 3 d\end{array}$ & $\begin{array}{l}{ }^{3} \mathrm{P}_{0}^{\mathrm{o}}(97.2 \%) \\
{ }^{3} \mathrm{P}_{1}^{\mathrm{o}}(96.9 \%) \\
{ }^{3} \mathrm{P}_{2}^{\mathrm{o}}(96.4 \%)\end{array}$ & $\begin{array}{l}3.36224 \\
3.37921 \\
3.41384\end{array}$ & $\begin{array}{l}3.40502 \\
3.42372 \\
3.46275\end{array}$ & $\begin{array}{l}3.19510 \\
3.21381 \\
3.25264\end{array}$ & $\begin{array}{l}{ }^{3} \mathrm{P}_{0}^{\mathrm{o}}(97.4 \%) \\
{ }^{3} \mathrm{P}_{1}^{\mathrm{o}}(96.9 \%) \\
{ }^{3} \mathrm{P}_{2}^{\mathrm{o}}(96.2 \%)\end{array}$ & $\begin{array}{l}3.67368 \\
3.69563 \\
3.74058\end{array}$ & $\begin{array}{l}3.69767 \\
3.72084 \\
3.76963\end{array}$ & $\begin{array}{l}3.45516 \\
3.47859 \\
3.52725\end{array}$ & $\begin{array}{l}3.69766 \\
3.72078 \\
3.76962\end{array}$ & $\begin{array}{l}3.76364 \\
3.78883 \\
3.84039\end{array}$ & $\begin{array}{l}3.7475 \\
3.7710 \\
3.8200\end{array}$ \\
\hline $\begin{array}{l}5 \\
6 \\
7\end{array}$ & $\begin{array}{l}3 s^{2} 3 p^{5} 3 d \\
3 s^{2} 3 p^{5} 3 d \\
3 s^{2} 3 p^{5} 3 d\end{array}$ & $\begin{array}{l}{ }^{3} \mathrm{~F}_{4}^{o}(97.5 \%) \\
{ }^{3} \mathrm{~F}_{3}^{\mathrm{o}}(94.9 \%) \\
{ }^{3} \mathrm{~F}_{2}^{\mathrm{o}}(95.5 \%)\end{array}$ & $\begin{array}{l}3.55446 \\
3.57113 \\
3.59269\end{array}$ & $\begin{array}{l}3.57067 \\
3.59878 \\
3.63198\end{array}$ & $\begin{array}{l}3.36662 \\
3.39298 \\
3.42463\end{array}$ & $\begin{array}{l}{ }^{3} \mathrm{~F}_{4}^{o}(97.6 \%) \\
{ }^{3} \mathrm{~F}_{3}^{o}(93.9 \%) \\
{ }^{3} \mathrm{~F}_{2}^{o}(94.6 \%)\end{array}$ & $\begin{array}{l}3.88447 \\
3.90343 \\
3.93169\end{array}$ & $\begin{array}{l}3.88026 \\
3.91217 \\
3.95325\end{array}$ & & $\begin{array}{l}3.88027 \\
3.91217 \\
3.95329\end{array}$ & $\begin{array}{l}3.97113 \\
4.00201 \\
4.04308\end{array}$ & $\begin{array}{l}3.9468 \\
3.9797 \\
4.0215\end{array}$ \\
\hline 8 & $3 s^{2} 3 p^{5} 3 d$ & ${ }^{3} \mathrm{D}_{3}^{\mathrm{o}}(68.7 \%)$ & 3.81841 & 3.82162 & 3.62227 & ${ }^{3} \mathrm{D}_{3}^{\mathrm{o}}(64.5 \%)$ & 4.16736 & 4.15184 & 3.92030 & 4.15171 & 4.25326 & 4.2373 \\
\hline 9 & $3 s^{2} 3 p^{5} 3 d$ & ${ }^{1} \mathrm{D}_{2}^{\mathrm{o}}(64.4 \%)$ & 3.81303 & 3.82565 & 3.62467 & ${ }^{1} \mathrm{D}_{2}^{\mathrm{o}}(62.1 \%)$ & 4.16451 & 4.16224 & 3.92846 & 4.16220 & 4.26847 & 4.2540 \\
\hline $\begin{array}{l}10 \\
11\end{array}$ & $\begin{array}{l}3 s^{2} 3 p^{5} 3 d \\
3 s^{2} 3 p^{5} 3 d\end{array}$ & $\begin{array}{l}{ }^{3} \mathrm{D}_{1}^{\mathrm{o}}(96.8 \%) \\
{ }^{3} \mathrm{D}_{2}^{\mathrm{o}}(64.7 \%)\end{array}$ & $\begin{array}{l}3.83602 \\
3.85013\end{array}$ & $\begin{array}{l}3.85773 \\
3.86961\end{array}$ & $\begin{array}{l}3.65585 \\
3.66842\end{array}$ & $\begin{array}{l}{ }^{3} \mathrm{D}_{1}^{\mathrm{o}}(96.8 \%) \\
{ }^{3} \mathrm{D}_{2}^{\mathrm{o}}(62.6 \%)\end{array}$ & $\begin{array}{l}4.19143 \\
4.21227\end{array}$ & $\begin{array}{l}4.19744 \\
4.21567\end{array}$ & $\begin{array}{l}3.96326 \\
3.98226\end{array}$ & $\begin{array}{l}4.19745 \\
4.21573\end{array}$ & $\begin{array}{l}4.29702 \\
4.31956\end{array}$ & $\begin{array}{l}4.2836 \\
4.3039\end{array}$ \\
\hline 12 & $3 s^{2} 3 p^{5} 3 d$ & ${ }^{\mathrm{l}} \mathrm{F}_{3}^{\mathrm{o}}(67.1 \%)$ & 3.86567 & 3.89595 & 3.69578 & ${ }^{1} \mathrm{~F}_{3}^{\mathrm{o}}(62.2 \%)$ & 4.22812 & 4.24494 & 4.01241 & 4.24509 & 4.34420 & 4.3281 \\
\hline 13 & $3 s^{2} 3 p^{5} 3 d$ & ${ }^{1} \mathrm{P}_{1}^{\mathrm{o}}(95.7 \%)^{\star}$ & 4.91368 & 4.91368 & 4.76446 & ${ }^{1} \mathrm{P}_{1}^{\mathrm{o}}(95.9 \%)^{\star}$ & 5.32660 & 5.32678 & $\begin{array}{r}5.14621 \\
5.212^{\mathrm{d}}\end{array}$ & 5.32645 & 5.47936 & 5.4598 \\
\hline 14 & $3 s 3 p^{6} 3 d$ & ${ }^{3} \mathrm{D}_{1}(73.5 \%)$ & 6.05426 & 6.08432 & 5.99785 & ${ }^{3} \mathrm{D}_{1}(74.7 \%)$ & 6.61242 & 6.62249 & 6.50906 & 6.62249 & 6.75891 & 6.7176 \\
\hline 15 & $3 s 3 p^{6} 3 d$ & ${ }^{3} \mathrm{D}_{2}(73.4 \%)$ & 6.06173 & 6.09007 & 6.00326 & ${ }^{3} \mathrm{D}_{2}(74.6 \%)$ & 6.62228 & 6.63001 & 6.51629 & 6.63001 & 6.76762 & 6.7251 \\
\hline 16 & $3 s 3 p^{6} 3 d$ & ${ }^{3} \mathrm{D}_{3}(73.7 \%)$ & 6.07394 & 6.09935 & 6.01167 & ${ }^{3} \mathrm{D}_{3}(74.9 \%)$ & 6.63882 & 6.64254 & 6.52770 & 6.64254 & 6.78194 & 6.7377 \\
\hline 17 & $3 \mathrm{~s} 3 \mathrm{p}^{6} 3 \mathrm{~d}$ & ${ }^{1} \mathrm{D}_{2}(68.2 \%)$ & 6.26561 & 6.27726 & 6.23324 & ${ }^{1} \mathrm{D}_{2}(69.4 \%)$ & 6.84122 & 6.83333 & 6.76228 & 6.83334 & 6.98165 & 6.9447 \\
\hline
\end{tabular}

Notes. ${ }^{(a)}$ present work: 2894-level MCBP results; ${ }^{(b)}$ critically compiled experimental data of Shirai et al. (2000); ${ }^{(c)}$ MCHF results of FroeseFischer \& Tachiev (2006); $\left.{ }^{\star}{ }^{\star}\right)$ dominant excitation threshold - see Table 2; ${ }^{(d)}$ single configuration TDCHF results of Ghosh \& Mukherjee (1997); ${ }^{(e)}$ CIV3 results of Verma et al. (2006); ${ }^{(f)}$ IRON Project results of Storey et al. (2002); ${ }^{(g)}$ 6284-level FAC results of Aggarwal et al. (2006);

(v08.00; Ferland et al. 1998). Although these results did not include the DR rate coefficient data reported here, the indicated temperature range should still be reasonably accurate.

For K II and Ca III, the only other data available in Fig. 1 are the results of Mewe et al. (1980) and Mazzotta et al. (1998). The rate coefficients of Mazzotta et al. (1998) are inferred by using the general formula of Burgess (1965) that is first scaled down to match the empirical DR rate of Hahn (1989) for Fe IX and then this same scaling is applied to other ions in the isoelectronic sequence. Surprisingly, this procedure gives results that are in fair agreement with our present calculations, but, as is seen for higher ionization stages, this method fails to account correctly for low-temperature DR. The results of Mewe et al. (1980), on the other hand, were obtained using a parameterized empirical formula based upon the renormalized results of Jacobs et al. (1977) and Ansari et al. (1970) for $3 p \rightarrow 3 d, 3 p \rightarrow 4 s$, and 
Table 6. The lowest $\Delta n_{\mathrm{c}}=0$ core excitation thresholds (in Rydbergs) for Co X and Ni XI. Uncertainties are enclosed in lower parentheses.

\begin{tabular}{|c|c|c|c|c|c|c|c|c|c|c|c|}
\hline \multirow[b]{2}{*}{$\mathrm{K}$} & \multirow[b]{2}{*}{ Config. } & \multicolumn{4}{|c|}{$\mathrm{Co}^{9+}$} & \multicolumn{6}{|c|}{$\mathrm{Ni}^{10+}$} \\
\hline & & Level(mix) & Present $^{a}$ & $\mathrm{NIST}^{b}$ & $\mathrm{MCHF}^{c}$ & Level(mix) & Present $^{a}$ & $\mathrm{NIST}^{b}$ & $\mathrm{MCHF}^{c}$ & $\mathrm{FAC}^{e}$ & CIV3 $^{f}$ \\
\hline 1 & $3 s^{2} 3 p^{6}$ & ${ }^{1} \mathrm{~S}_{0}(97.0 \%)$ & 0.00000 & 0.00000 & 0.00000 & ${ }^{1} \mathrm{~S}_{0}(97.1 \%)$ & 0.00000 & 0.00000 & 0.00000 & 0.0000 & 0.00000 \\
\hline $\begin{array}{l}2 \\
3 \\
4\end{array}$ & $\begin{array}{l}3 s^{2} 3 p^{5} 3 d \\
3 s^{2} 3 p^{5} 3 d \\
3 s^{2} 3 p^{5} 3 d\end{array}$ & $\begin{array}{l}{ }^{3} \mathrm{P}_{0}^{\mathrm{o}}(97.5 \%) \\
{ }^{3} \mathrm{P}_{1}^{\mathrm{o}}(96.9 \%) \\
{ }^{3} \mathrm{P}_{2}^{\mathrm{o}}(95.8 \%)\end{array}$ & $\begin{array}{l}3.97623 \\
4.00373 \\
4.06022\end{array}$ & $\begin{array}{l}3.999_{(30)}^{\dagger} \\
4.026_{(28)}^{\dagger} \\
4.084_{(21)}^{\dagger}\end{array}$ & $\begin{array}{l}3.70893 \\
3.73778 \\
3.79773\end{array}$ & $\begin{array}{l}{ }^{3} \mathrm{P}_{0}^{\mathrm{o}}(97.6 \%) \\
{ }^{3} \mathrm{P}_{1}^{\mathrm{o}}(96.8 \%) \\
{ }^{3} \mathrm{P}_{2}^{\mathrm{o}}(95.2 \%)\end{array}$ & $\begin{array}{l}4.27882 \\
4.31288 \\
4.38307\end{array}$ & $\begin{array}{l}4.27667 \\
4.31002 \\
4.38274\end{array}$ & $\begin{array}{l}3.95697 \\
3.99197 \\
4.06469\end{array}$ & $\begin{array}{l}4.2998 \\
4.3349 \\
4.4086\end{array}$ & $\begin{array}{l}4.34736 \\
4.38366 \\
4.45723\end{array}$ \\
\hline $\begin{array}{l}5 \\
6 \\
7\end{array}$ & $\begin{array}{l}3 s^{2} 3 p^{5} 3 d \\
3 s^{2} 3 p^{5} 3 d \\
3 s^{2} 3 p^{5} 3 d\end{array}$ & $\begin{array}{l}{ }^{3} \mathrm{~F}_{4}^{\mathrm{o}}(97.7 \%) \\
{ }^{3} \mathrm{~F}_{3}^{\mathrm{o}}(92.8 \%) \\
{ }^{3} \mathrm{~F}_{2}^{\mathrm{o}}(93.2 \%)\end{array}$ & $\begin{array}{l}4.20594 \\
4.22715 \\
4.26350\end{array}$ & $\begin{array}{l}4.199_{(31)}^{\dagger} \\
4.235_{(31)}^{\dagger} \\
4.283_{(25)}^{\dagger}\end{array}$ & $\begin{array}{l}3.91676 \\
3.94997 \\
3.99798\end{array}$ & $\begin{array}{l}{ }^{3} \mathrm{~F}_{4}^{\mathrm{o}}(97.8 \%) \\
{ }^{3} \mathrm{~F}_{3}^{\mathrm{o}}(91.3 \%) \\
{ }^{3} \mathrm{~F}_{2}^{\mathrm{o}}(91.3 \%)\end{array}$ & $\begin{array}{l}4.52944 \\
4.55153 \\
4.59721\end{array}$ & $\begin{array}{l}4.49482 \\
4.53374 \\
4.59342\end{array}$ & $\begin{array}{l}4.18448 \\
4.22023 \\
4.27778\end{array}$ & $\begin{array}{l}4.5391 \\
4.5785 \\
4.6393\end{array}$ & $\begin{array}{l}4.64639 \\
4.68656 \\
4.73636\end{array}$ \\
\hline 8 & $3 s^{2} 3 p^{5} 3 d$ & ${ }^{3} \mathrm{D}_{3}^{\mathrm{o}}(62.3 \%)$ & 4.50743 & $4.494_{(38)}^{\dagger}$ & 4.21215 & ${ }^{3} \mathrm{D}_{3}^{\mathrm{o}}(60.3 \%)$ & 4.84888 & 4.80666 & 4.49868 & 4.8593 & 4.93579 \\
\hline 9 & $3 s^{2} 3 p^{5} 3 d$ & ${ }^{1} \mathrm{D}_{2}^{\mathrm{o}}(60.5 \%)$ & 4.50846 & $4.511_{(29)}^{\dagger}$ & 4.22855 & ${ }^{1} \mathrm{D}_{2}^{\mathrm{o}}(58.8 \%)$ & 4.85545 & 4.83728 & 4.52643 & 4.8953 & 4.97061 \\
\hline $\begin{array}{l}10 \\
11\end{array}$ & $\begin{array}{l}3 s^{2} 3 p^{5} 3 d \\
3 s^{2} 3 p^{5} 3 d\end{array}$ & $\begin{array}{l}{ }^{3} \mathrm{D}_{1}^{\mathrm{o}}(96.7 \%) \\
{ }^{3} \mathrm{D}_{2}^{\mathrm{o}}(61.4 \%)\end{array}$ & $\begin{array}{l}4.53943 \\
4.56821\end{array}$ & $\begin{array}{r}4.53611 \\
4.573_{(24)}^{\dagger}\end{array}$ & $\begin{array}{l}4.26634 \\
4.29382\end{array}$ & $\begin{array}{l}{ }^{3} \mathrm{D}_{1}^{\mathrm{o}}(96.5 \%) \\
{ }^{3} \mathrm{D}_{2}^{\mathrm{o}}(60.2 \%)\end{array}$ & $\begin{array}{l}4.88993 \\
4.92948\end{array}$ & $\begin{array}{l}4.87373 \\
4.91337\end{array}$ & $\begin{array}{l}4.56612 \\
4.60454\end{array}$ & $\begin{array}{l}4.9290 \\
4.9687\end{array}$ & $\begin{array}{l}5.02599 \\
5.04846\end{array}$ \\
\hline 12 & $3 s^{2} 3 p^{5} 3 d$ & ${ }^{1} \mathrm{~F}_{3}^{\mathrm{o}}(59.0 \%)$ & 4.58588 & $4.606_{(25)}^{\dagger}$ & 4.32726 & ${ }^{1} \mathrm{~F}_{3}^{\mathrm{o}}(56.1 \%)$ & 4.94891 & 4.95018 & 4.64195 & 5.0024 & 5.12673 \\
\hline 13 & $3 s^{2} 3 p^{5} 3 d$ & ${ }^{1} \mathrm{P}_{1}^{\mathrm{o}}(96.1 \%)^{\star}$ & 5.73579 & 5.73579 & $\begin{array}{l}5.51933 \\
5.5952^{\mathrm{d}}\end{array}$ & ${ }^{1} \mathrm{P}_{1}^{\mathrm{o}}(96.2 \%)^{\star}$ & 6.14044 & 6.14158 & $\begin{array}{l}5.88618 \\
5.9728^{d}\end{array}$ & 6.2574 & 6.41884 \\
\hline $\begin{array}{l}14 \\
15 \\
16\end{array}$ & $\begin{array}{l}3 s 3 p^{6} 3 d \\
3 s 3 p^{6} 3 d \\
3 s 3 p^{6} 3 d\end{array}$ & $\begin{array}{l}{ }^{3} \mathrm{D}_{1}(74.2 \%) \\
{ }^{3} \mathrm{D}_{2}(74.0 \%) \\
{ }^{3} \mathrm{D}_{3}(74.4 \%)\end{array}$ & $\begin{array}{l}7.21441 \\
7.22694 \\
7.24842\end{array}$ & $\begin{array}{l}7.178_{(23)}^{\dagger} \\
7.186_{(31)}^{\dagger} \\
7.198_{(31)}^{\dagger}\end{array}$ & $\begin{array}{l}7.01984 \\
7.02926 \\
7.04436\end{array}$ & $\begin{array}{l}{ }^{3} \mathrm{D}_{1}(75.1 \%) \\
{ }^{3} \mathrm{D}_{2}(74.1 \%) \\
{ }^{3} \mathrm{D}_{3}(75.3 \%)\end{array}$ & $\begin{array}{l}7.76928 \\
7.78502 \\
7.81290\end{array}$ & $\begin{array}{l}7.720_{(31)}^{\dagger} \\
7.730_{(32)}^{\dagger} \\
7.740_{(33)}{ }^{\dagger}\end{array}$ & $\begin{array}{l}7.53153 \\
7.54354 \\
7.56317\end{array}$ & $\begin{array}{l}7.7562 \\
7.7687 \\
7.7904\end{array}$ & $\begin{array}{l}7.85347 \\
7.86852 \\
7.89090\end{array}$ \\
\hline 17 & $3 s 3 p^{6} 3 d$ & ${ }^{1} \mathrm{D}_{2}(68.8 \%)$ & 7.46032 & $7.410_{(33)}^{\dagger}$ & 7.29060 & ${ }^{1} \mathrm{D}_{2}(69.7 \%)$ & 8.03277 & $7.970_{(34)}^{\dagger}$ & 7.81976 & 7.9965 & 9.00447 \\
\hline
\end{tabular}

Notes. ${ }^{(a)}$ present work: 2894-level MCBP results; ${ }^{(b)}$ critically compiled experimental data of Shirai et al. (2000); ${ }^{(c)}$ MCHF results of FroeseFischer \& Tachiev (2006); ${ }^{(\dagger)}$ extrapolated along the isoelectronic sequence; $\left.{ }^{\star}{ }^{\star}\right)$ dominant excitation threshold - see Table 2; ${ }^{(d)}$ single-configuration TDCHF results of Ghosh \& Mukherjee (1997); ${ }^{(e)} 6164-$ level FAC results of Aggarwal \& Keenan $(2008) ;{ }^{(f)}$ restricted CIV3 results of Verma et al. (2007).

Table 7. The lowest $\Delta n_{\mathrm{c}}=0$ core excitation thresholds (in Rydbergs) for Cu XII and Zn XIII. Uncertainties are enclosed in lower parentheses.

\begin{tabular}{|c|c|c|c|c|c|c|c|c|c|}
\hline \multirow[b]{2}{*}{ K } & \multirow[b]{2}{*}{ Config. } & \multicolumn{4}{|c|}{$\mathrm{Cu}^{\mathrm{II+}}$} & \multicolumn{4}{|c|}{$\overline{\mathrm{Zn}^{12+}}$} \\
\hline & & Level(mix) & Present $^{a}$ & NIST $^{b}$ & $\mathrm{MCHF}^{c}$ & Level(mix) & Present $^{a}$ & $\mathrm{NIST}^{b}$ & $\mathrm{MCHF}^{c}$ \\
\hline 1 & $3 s^{2} 3 p^{6}$ & ${ }^{\mathrm{T}} \mathrm{S}_{0}(97.2 \%)$ & 0.000000 & 0.000000 & 0.000000 & ${ }^{\mathrm{T}} \mathrm{S}_{0}(97.3 \%)$ & 0.00000 & 0.00000 & 0.00000 \\
\hline 2 & $3 s^{2} 3 p^{5} 3 d$ & ${ }^{3} \mathrm{P}_{0}^{\mathrm{o}}(97.7 \%)$ & 4.56676 & $4.603_{(36)}^{\S}$ & 4.19956 & ${ }^{3} \mathrm{P}_{0}^{\mathrm{o}}(97.7 \%)$ & 4.85185 & $4.905_{(38)}{ }^{\S}$ & 4.43697 \\
\hline 3 & $3 s^{2} 3 p^{5} 3 d$ & ${ }^{3} \mathrm{P}_{1}^{0}(96.6 \%)$ & 4.60819 & $4.638_{(32)} \S$ & 4.24146 & ${ }^{3} \mathrm{P}_{1}^{0}(96.3 \%)$ & 4.90147 & $4.943_{(35)} \S$ & 4.48650 \\
\hline 4 & $3 s^{2} 3 p^{5} 3 d$ & ${ }^{3} \mathrm{P}_{2}^{\mathrm{o}}(94.4 \%)$ & 4.69378 & $4.711_{(25)} \S$ & 4.32844 & ${ }^{3} \mathrm{P}_{2}^{\mathrm{o}}(93.4 \%)$ & 5.00416 & $5.025_{(27)} \S$ & 4.58924 \\
\hline 5 & $3 s^{2} 3 p^{5} 3 d$ & ${ }^{3} \mathrm{~F}_{4}^{\mathrm{o}}(97.9 \%)$ & 4.83899 & $4.837_{(36)}^{\S}$ & 4.44807 & ${ }^{3} \mathrm{~F}_{4}^{\mathrm{o}}(97.9 \%)$ & 5.14710 & $5.156_{(38)} \S$ & 4.70801 \\
\hline 6 & $3 s^{2} 3 p^{5} 3 d$ & ${ }^{3} \mathrm{~F}_{3}^{\mathrm{o}}(89.6 \%)$ & 4.86273 & $4.880_{(35)} \S$ & 4.48537 & ${ }^{3} \mathrm{~F}_{3}^{\mathrm{o}}(87.7 \%)$ & 5.17130 & $5.203_{(38)}{ }^{\S}$ & 4.74554 \\
\hline 7 & $3 s^{2} 3 p^{5} 3 d$ & ${ }^{3} \mathrm{~F}_{2}^{\mathrm{o}}(88.5 \%)$ & 4.92015 & $4.941_{(29)} \S$ & 4.55309 & ${ }^{3} \mathrm{~F}_{2}^{\mathrm{o}}(84.9 \%)$ & 5.24156 & $5.270_{(31)}^{\S}$ & 4.82380 \\
\hline 8 & $3 s^{2} 3 p^{5} 3 d$ & ${ }^{1} \mathrm{D}_{2}^{\mathrm{o}}(57.2 \%)$ & 5.19405 & $5.203_{(34)}^{\S}$ & 4.82348 & ${ }^{1} \mathrm{D}_{2}^{\mathrm{o}}(55.4 \%)$ & 5.53462 & $5.548_{(36)} \S$ & 5.12106 \\
\hline 9 & $3 s^{2} 3 p^{5} 3 d$ & ${ }^{3} \mathrm{D}_{3}^{\mathrm{o}}(59.8 \%)$ & 5.17774 & $5.177_{(44)} \S$ & 4.78044 & ${ }^{3} \mathrm{D}_{3}^{\mathrm{o}}(59.6 \%)$ & 5.50490 & $5.519_{(47)} \S$ & 5.05792 \\
\hline 10 & $3 s^{2} 3 p^{5} 3 d$ & ${ }^{3} \mathrm{D}_{1}^{\mathrm{o}}(96.2 \%)$ & 5.23130 & 5.21500 & 4.86330 & ${ }^{3} \mathrm{D}_{1}^{\mathrm{o}}(95.8 \%)$ & 5.57297 & 5.55700 & 5.15855 \\
\hline 11 & $3 s^{2} 3 p^{5} 3 d$ & ${ }^{3} \mathrm{D}_{2}^{\mathrm{o}}(59.4 \%)$ & 5.28354 & $5.280_{(32)} \S$ & 4.91565 & ${ }^{3} \mathrm{D}_{2}^{\mathrm{o}}(58.7 \%)$ & 5.64097 & $5.633_{(35)}{ }^{\S}$ & 5.22837 \\
\hline 12 & $3 s^{2} 3 p^{5} 3 d$ & ${ }^{1} \mathrm{~F}_{3}^{2}(54.3 \%)$ & 5.30733 & $5.320_{(29)} \S$ & 4.95778 & ${ }^{1} \mathrm{~F}_{3}^{\mathrm{o}}(52.7 \%)$ & 5.67000 & $5.676_{(31)} 8$ & 5.27605 \\
\hline 13 & $3 s^{2} 3 p^{5} 3 d$ & ${ }^{1} \mathrm{P}_{1}^{\mathrm{o}}(96.2 \%)^{\star}$ & 6.54764 & 6.54764 & $\begin{array}{r}6.24830 \\
6.346^{\mathrm{d}}\end{array}$ & ${ }^{1} \mathrm{P}_{1}^{\mathrm{o}}(96.1 \%)^{\star}$ & 6.95380 & 6.95379 & $\begin{array}{l}6.60700 \\
6.7154^{\mathrm{d}}\end{array}$ \\
\hline 14 & $3 s 3 p^{6} 3 d$ & ${ }^{3} \mathrm{D}_{1}(75.6 \%)$ & 8.29610 & $8.268_{(27)} \S$ & 8.04518 & ${ }^{3} \mathrm{D}_{1}(75.9 \%)$ & 8.82751 & $8.814_{(29)} \S$ & 8.56194 \\
\hline 15 & $3 s 3 p^{6} 3 d$ & ${ }^{3} \mathrm{D}_{2}(74.9 \%)$ & 8.31511 & $8.277_{(36)}{ }^{\S}$ & 8.06020 & ${ }^{3} \mathrm{D}_{2}(75.1 \%)$ & 8.85018 & $8.822_{(38)}{ }^{8}$ & 8.58042 \\
\hline 16 & $3 s 3 p^{6} 3 d$ & ${ }^{3} \mathrm{D}_{3}(75.9 \%)$ & 8.34975 & $8.291_{(36)} \S$ & 8.08529 & ${ }^{3} \mathrm{D}_{3}(76.3 \%)$ & 8.89283 & $8.838_{(39)}^{\S}$ & 8.61207 \\
\hline 17 & $3 s 3 p^{6} 3 d$ & ${ }^{1} \mathrm{D}_{2}(70.1 \%)$ & 8.57720 & $8.538_{(39)}{ }^{\S}$ & 8.35116 & ${ }^{1} \mathrm{D}_{2}(70.3 \%)$ & 9.12726 & $9.102_{(42)}{ }^{8}$ & 8.88624 \\
\hline
\end{tabular}

Notes. ${ }^{(a)}$ present work: 2894-level MCBP results; ${ }^{(b)}$ critically compiled experimental data of Shirai et al. $(2000) ;{ }^{(c)}$ MCHF results of FroeseFischer \& Tachiev (2006); ${ }^{(\S)}$ extrapolated along the isoelectronic sequence; ${ }^{\star}{ }^{()}$dominant excitation threshold - see Table 2; ${ }^{(d)}$ single configuration TDCHF results of Ghosh \& Mukherjee (1997).

$3 p \rightarrow 4 d$ core-excitations of Fe IX; that procedure gives erroneous results for the position and peak of the rate coefficient.

The results shown in Fig. 2 have been analyzed more fully in our earlier studies of Sc IV (Nikolić et al. 2010) and Ti V (Nikolić et al. 2009), but the following points should be made. First, there is experimental data available for both of these ions (Schippers et al. 1998, 2002), and these measurements were useful for quantifying the positions of low-energy resonances. However, the experiments for these low-charged Ar-like ions were subject to motional Stark effect that reionized the higher- $n$ recombined bound 

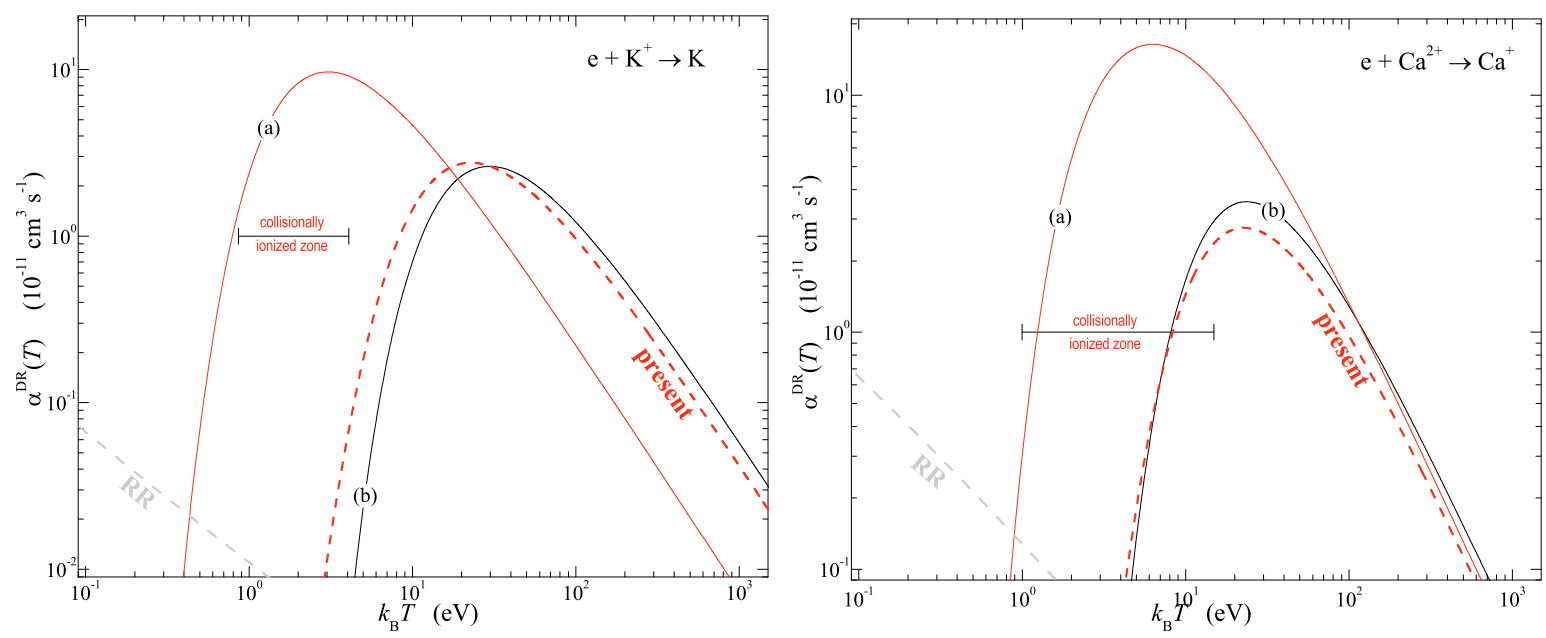

Fig. 1. Comparison of existing total DR ground-level rate coefficients for K II (left) and Ca III (right): (a) red solid curve, empirical results of Mewe et al. (1980); (b) black solid curve, recommended data of Mazzotta et al. (1998); red dashed curve, present MCBP results. RR channels are also shown as the long-dashed curve.
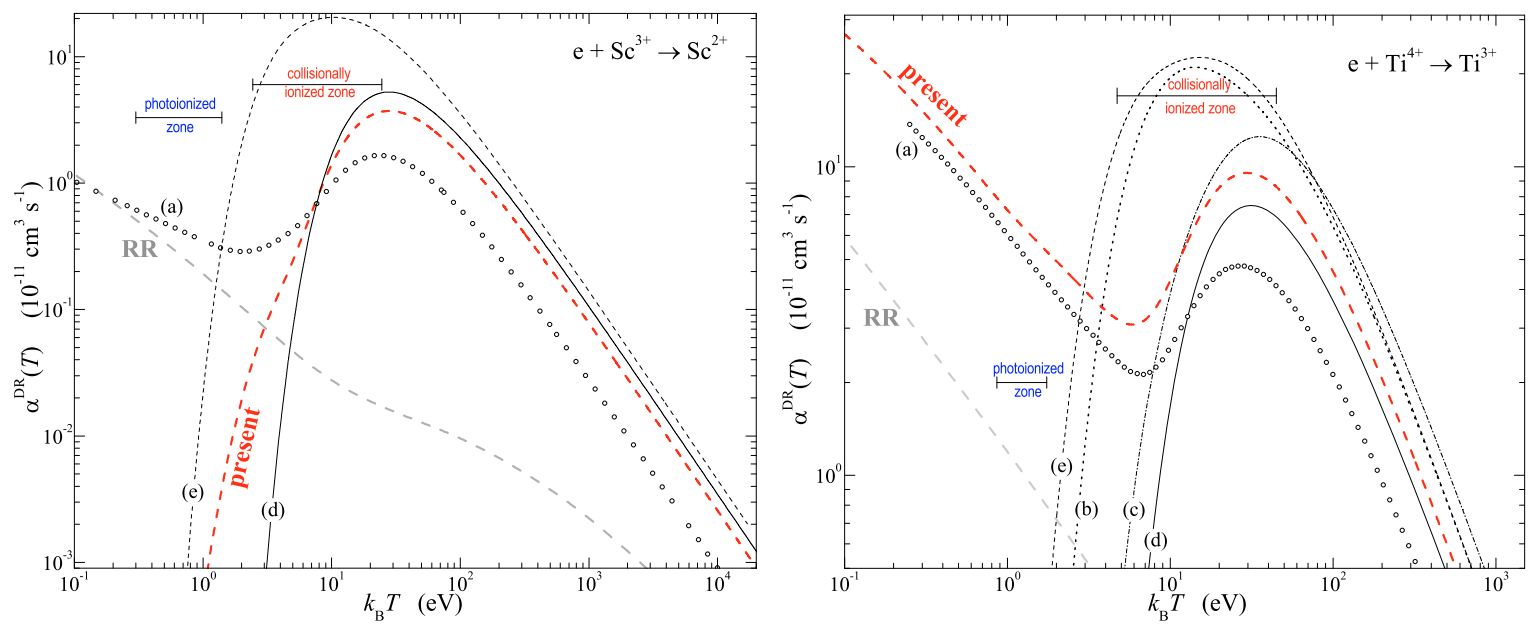

Fig. 2. Comparison of existing total DR ground-level rate coefficients for Sc IV (left) and Ti V (right): (a) gray open circles, TSR experiments by Schippers et al. (1998, 2002); (b) black doted curve, compilation by Landini \& Fossi (1991); (c) black dash-dotted curve, empirical formula of Hahn (1991); (d) black solid curve, recommended value by Mazzotta et al. (1998); (e) black dashed curve, empirical results of Mewe et al. (1980). The present RR results are also shown as the long-dashed curve.

states and led to a reduction in the DR cross section. Thus, the measured rate coefficient is unphysically too low at higher temperatures and is inappropriate for use in plasma modeling. Also, the experimental rate coefficient also contains the contribution due to RR, which is why the Sc IV experimental rate coefficient exceeds our DR result at lower temperatures and merges with our RR result.

Another new feature seen in Fig. 2 that was absent in Fig. 1 is that, as the ionization stage is increased, the lowest-lying members of the Rydberg series given in Eq. (1), namely the $3 s^{2} 3 p^{5} 3 d^{2}$ and $3 s^{2} 3 p^{5} 3 d 4 s$ resonances, approach zero continuum energy and give a large contribution to the rate coefficient at the lower temperatures found in photoionized plasmas. This contribution is not included in the results of Mazzotta et al. (1998); those data are determined from the Burgess formula (Burgess 1965), which only considers high-temperature DR. We note that the data of Mazzotta et al. (1998) also differs significantly from the present DR rate coefficient in the collisionally ionized zone.
The other available data - the empirical results of Mewe et al. (1980), Landini \& Fossi (1991), and Hahn (1991) - differ dramatically from the present MCBP results.

For the higher ionization stages of V VI, Cr VII, and Mn VIII shown in Figs. 3 and 4, the present rate coefficient again has a significant low-temperature DR contribution that is absent in all previously available data. For Fe IX, there exist several other results, but most of these are again empirical results that fail to account for low-temperature DR contributions in the photoionized plasma region and also differ significantly from our present DR results in the collisionally-ionized plasma region. We also show the earlier MCBP results of Badnell (2006). Those calculations were quite similar to the present ones, except that a smaller configuration basis was used and thus a slightly larger rate coefficient was obtained. We have established that by augmenting those earlier calculations with the inclusion of additional correlation configurations, as we include here, the two results are brought into agreement. Both MCBP results are somewhat 

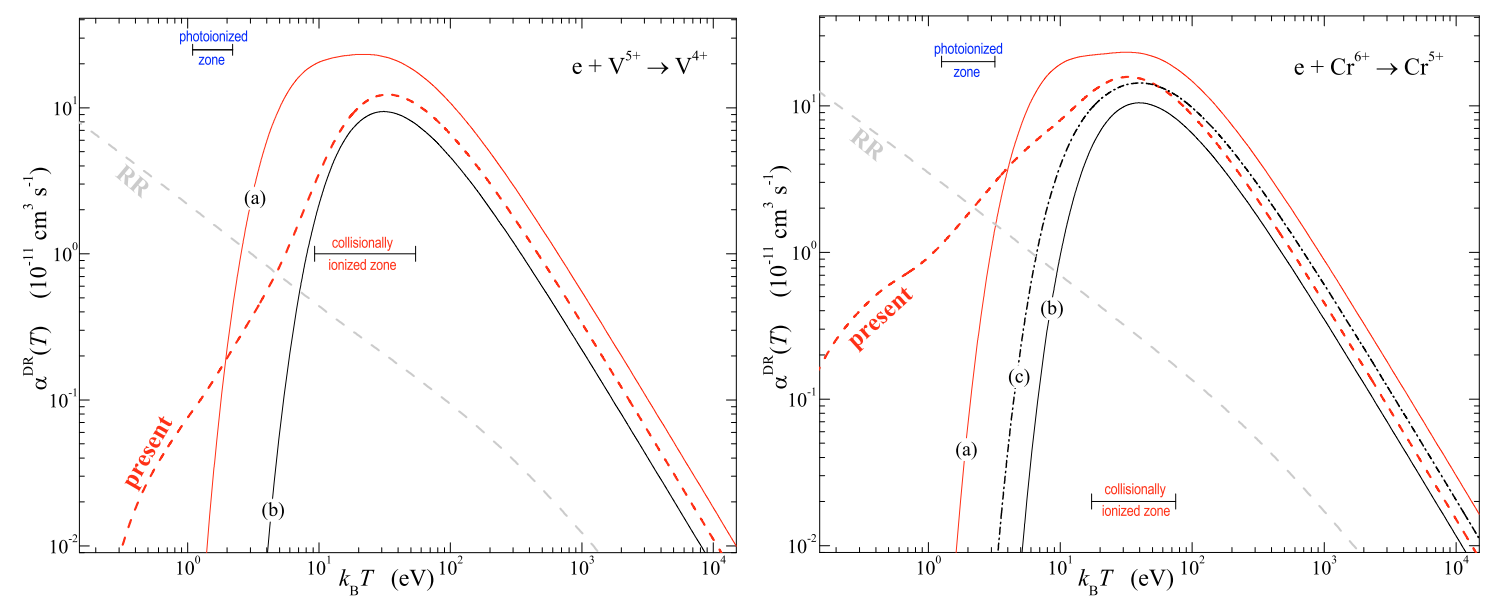

Fig. 3. Comparison of existing total DR ground-level rate coefficients for V VI (left) and Cr VII (right): (a) red solid curve, empirical results of Mewe et al. (1980); (b) black solid curve, recommended data of Mazzotta et al. (1998); (c) black dash-dotted curve, empirical formula of Hahn (1991); red dashed curve, present MCBP results. RR channels are also shown as the long-dashed curve.
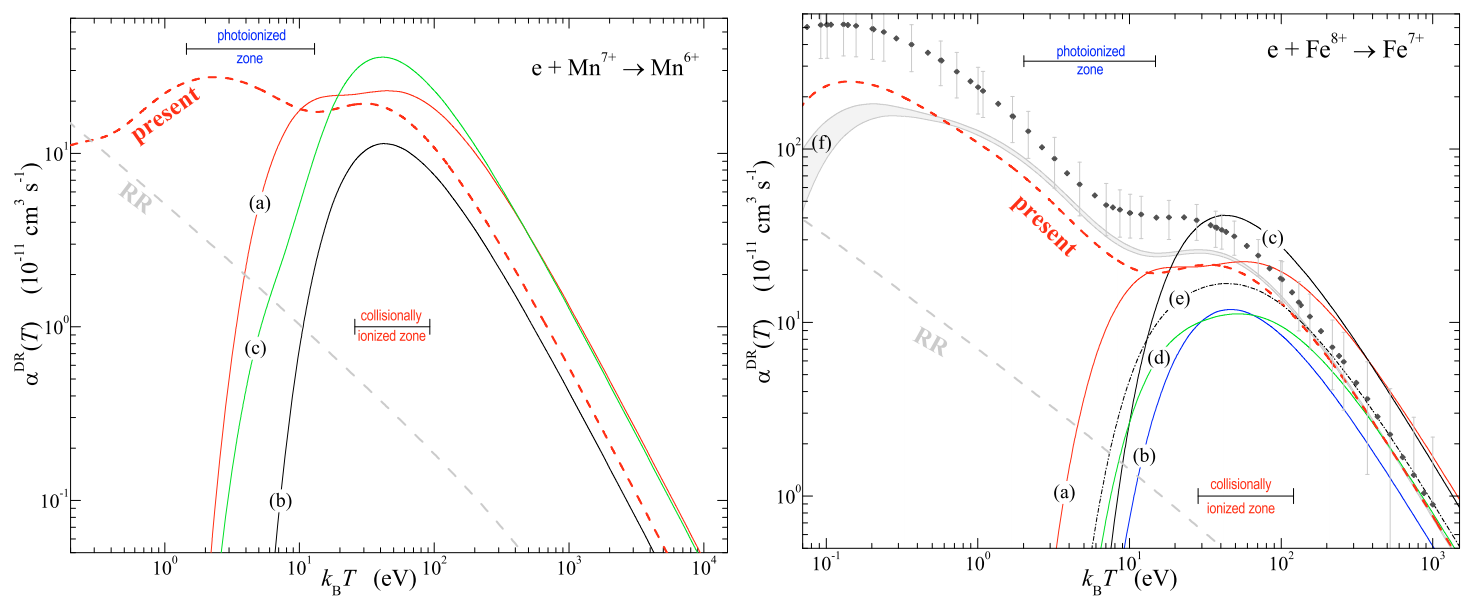

Fig. 4. Comparison of existing total DR ground-level rate coefficients. On the left is for Mn VIII: (a) red solid curve, empirical results of Mewe et al. (1980); (b) black solid curve, recommended data of Mazzotta et al. (1998); (c) green solid curve, LS results of Jacobs \& Davis (1983); red dashed curve, present MCBP results. On the right is Fe IX: black dots with error bars, TSR experiment by Schmidt et al. (2008); (a) red solid curve, empirical results of Mewe et al. (1980); (b) blue solid curve, compilation of Arnaud \& Raymond (1992); (c) black solid curve, result of Jacobs et al. (1977); (d) green solid curve, empirical data of Kato \& Asano (1999); (e) black dash-dotted curve, empirical formula of Hahn (1991); (f) "4CF" and "5CF" MCBP results of Badnell (2006); red dashed curve, present "18CF" MCBP results; The present RR results are also shown as the long-dashed curve.

lower than the experimental rate coefficient, as has been discussed more fully by Schmidt et al. (2008). We note that the experiment for Fe IX was not influenced by external fields, unlike the experiments for $\mathrm{Sc} \mathrm{IV}$ and $\mathrm{Ti} \mathrm{V}$, but may contain trielectronic recombination contributions, which are included in our computations as well. Our DR rate coefficients for Co X through Zn XIII are shown in Figs. 5 and 6, where it is seen again that all previous data do not account for the low-temperature contributions that dominate in the photoionized plasma region.

In Figs. 7-9, we show the DR contributions from each resonance series. As anticipated due to its large core oscillator strength, the $3 \mathrm{~s}^{2} 3 \mathrm{p}^{5} 3 \mathrm{~d} n \ell$ resonances are dominant. The lowest $3 \mathrm{~s}^{2} 3 \mathrm{p}^{5} 3 \mathrm{~d}^{2}$ resonances are first seen to be indistinct from the rest of the series for K II and Ca III, then begin to show a separate feature at low temperatures for Sc IV, and completely dominate at low temperatures for Ti V. As the nuclear charge is increased from V VI through Zn XIII, additional low-temperature features are seen to oscillate as the $n=4$ and $n=5$ resonances move from above to below threshold. Also seen as the ionization stage increases is the appearance of the $3 \mathrm{~s}^{2} 3 \mathrm{p}^{4} 3 \mathrm{~d}^{2} n \ell(n=3,4)$ resonances near threshold that dominate the low-temperature rate coefficient.

Lastly, we list the DR fitting coefficients for each ion, as described by Eq. (5), in Table 8. The RR fitting coefficients of Eq. (6) are listed in Table 9.

\section{Summary}

It is clear that all previously-available DR data for Ar-like ions are inadequate at lower temperatures since the contributions from low-lying resonances are not accounted for. Thus, those earlier data are inappropriate for use in modeling photoionized plasmas. And even at higher temperatures relevant to collisionally ionized plasmas, the earlier data were obtained using rather 
D. Nikolić et al.: Dielectronic recombination of argon-like ions
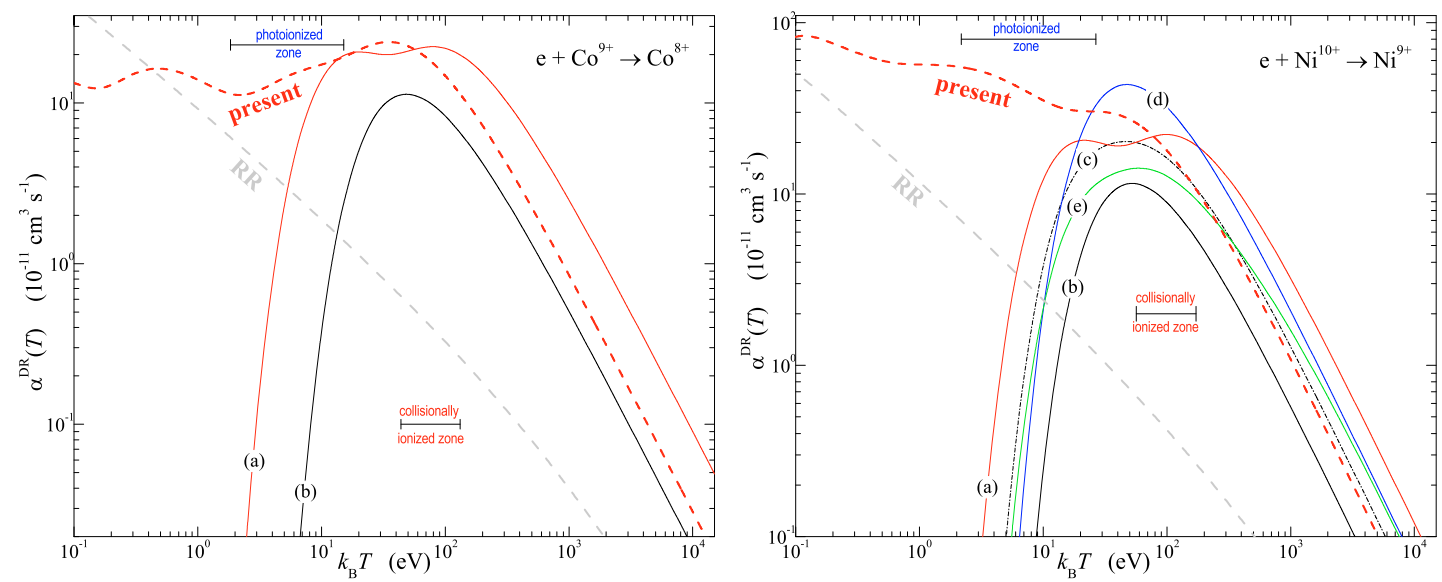

Fig. 5. Comparison of existing total DR ground-level rate coefficients. On the left is for Co X: (a) red solid curve, empirical results of Mewe et al. (1980); (b) black solid curve, recommended data of Mazzotta et al. (1998); red dashed curve, present MCBP results. On the right is for Ni XI ion: (a) red solid curve, empirical results of Mewe et al. (1980); (b) black solid curve, recommended data of Mazzotta et al. (1998); (c) black dash-doted curve, empirical formula of Hahn (1991); (d) blue solid curve, compilation of Arnaud \& Rothenflug (1985); (e) green solid curve, empirical data of Kato \& Asano (1999); red dashed curve, the present MCBP results. RR channels are also shown as the long-dashed curve.
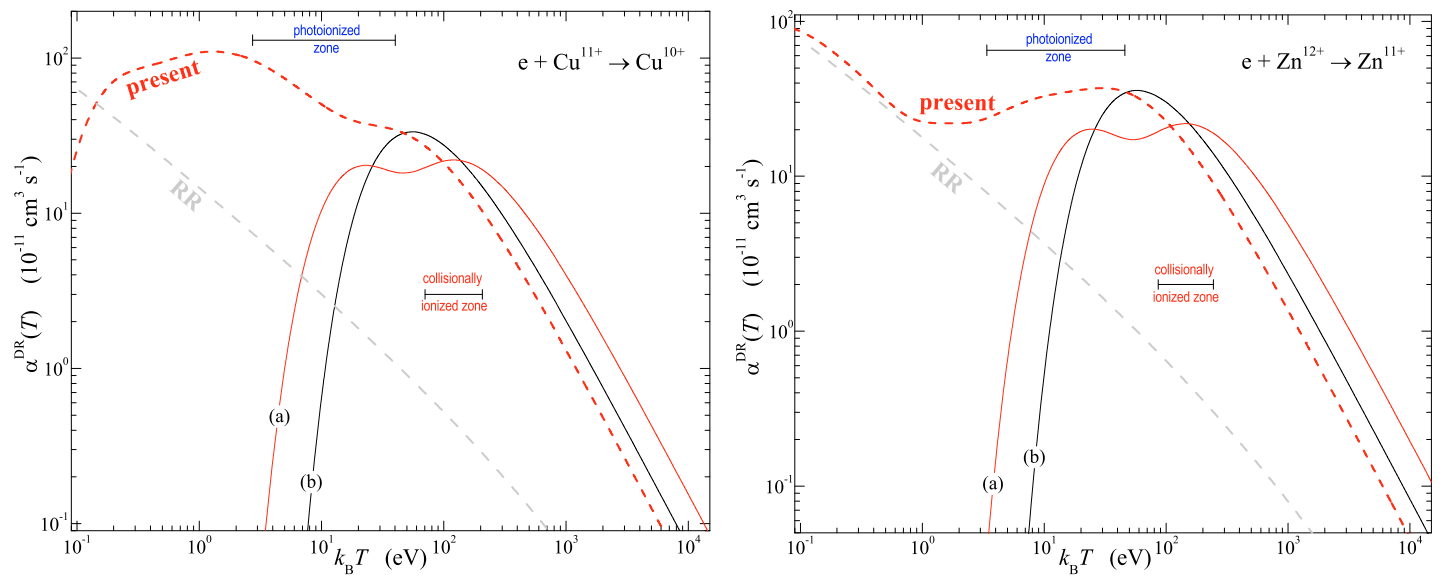

Fig. 6. Comparison of existing total DR ground-level rate coefficients for Cu XII (left) and Zn XIII (right): (a) red solid curve, empirical results of Mewe et al. (1980); (b) black solid curve, recommended data of Mazzitelli \& Mattioli (2002); red dashed curve, present MCBP results. RR channels are also shown as the long-dashed curve.
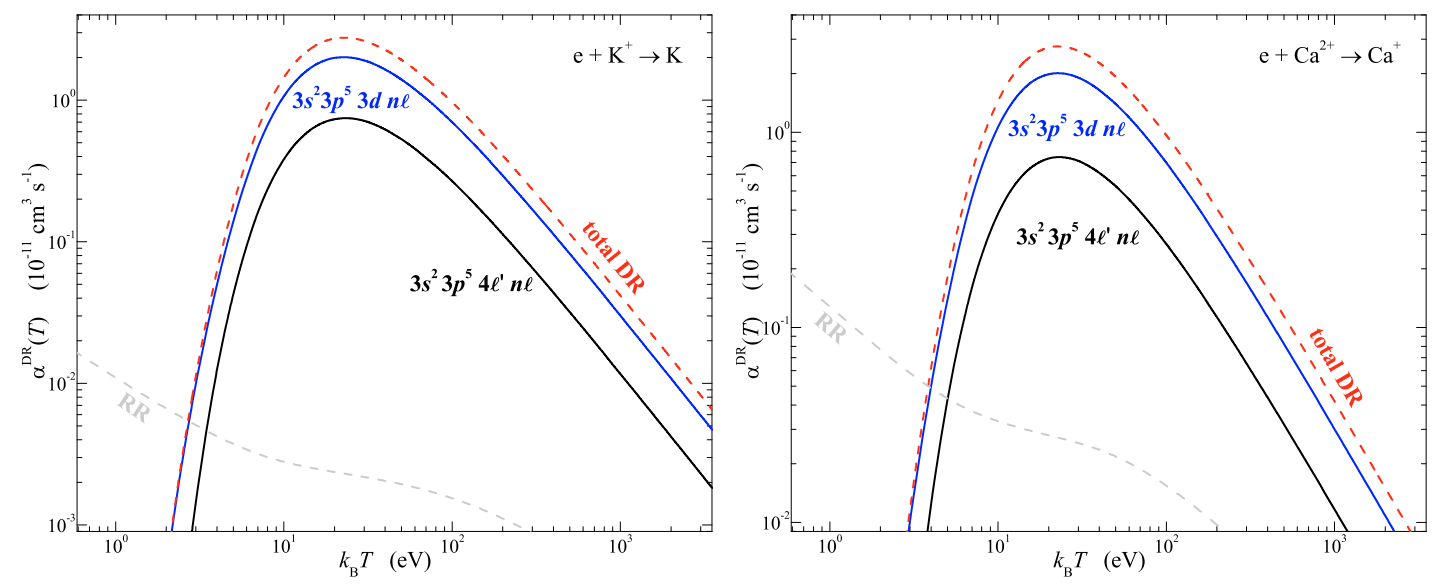

Fig. 7. Present Maxwellian-averaged DR and RR rate coefficients for K II (left $)$ and Ca III (right). The red and gray dashed curves are the total DR and RR rate coefficients, respectfully. The intra-shell $\Delta n_{\mathrm{c}}=0$ contributions (solid blue curve) are due to dominant $3 \mathrm{p} \rightarrow 3 \mathrm{~d}$ core excitations; the inter-shell $\Delta n_{\mathrm{c}}=1$ contributions (solid black curve) are due to $3 \mathrm{p} \rightarrow 4 \ell^{\prime}$ promotions. 

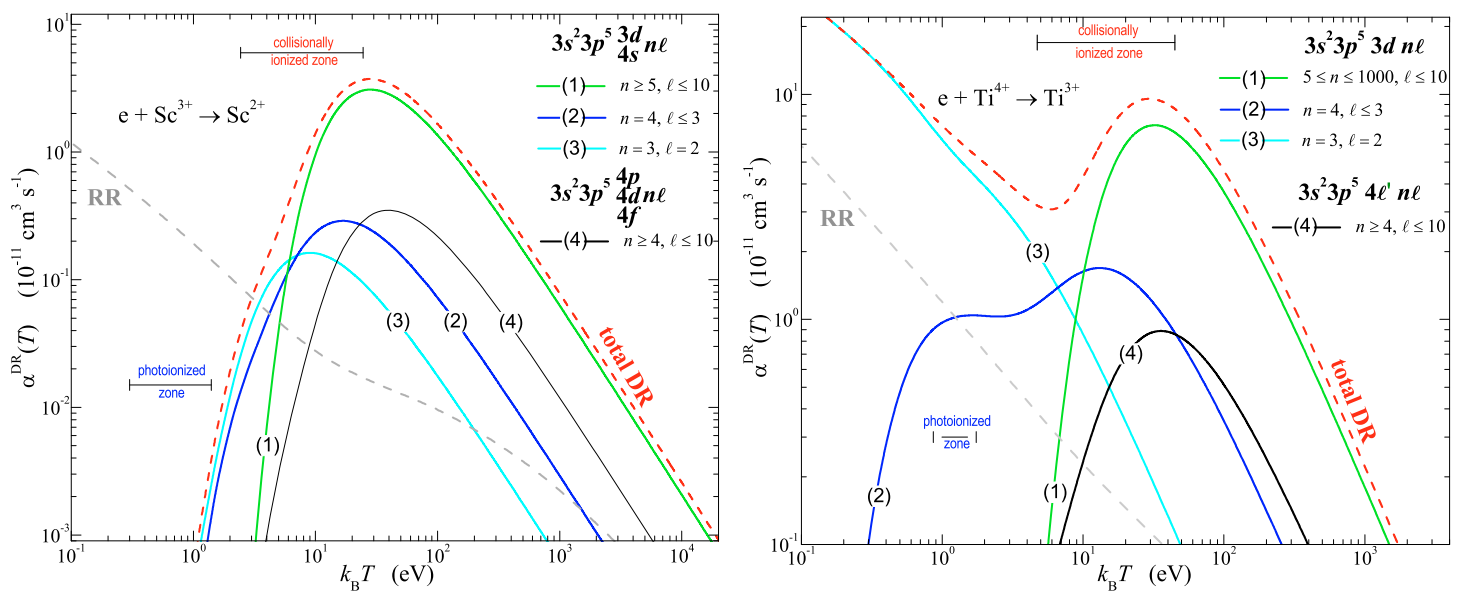

Fig. 8. Present Maxwellian-averaged DR and RR rate coefficients for Sc IV (left $)$ and Ti V (right). The red and gray dashed curves are the total DR and RR rate coefficients, respectfully. The intra-shell $\Delta n_{\mathrm{c}}=0$ contributions are due to dominant $3 \mathrm{p} \rightarrow 3 \mathrm{~d}$ core excitations; the inter-shell $\Delta n_{\mathrm{c}}=1$ contributions are due to $3 \mathrm{p} \rightarrow 4 \ell^{\prime}$ promotions.

Table 8. Fitting parameters $E_{i}(\mathrm{~K})$ and $c_{i}\left(\mathrm{~cm}^{3} \mathrm{~s}^{-1} \mathrm{~K}^{3 / 2}\right)$ used for modeling the Maxwellian-averaged DR rate coefficients - see Eq. (5). Uncertainties are enclosed in lower parentheses, where $v_{(u)}^{[ \pm p]}$ denotes $v(u) \times 10^{ \pm p}$.

\begin{tabular}{|c|c|c|c|c|c|c|c|c|c|c|c|c|c|c|}
\hline ion & $E_{1}$ & $E_{2}$ & $E_{3}$ & $E_{4}$ & $E_{5}$ & $E_{6}$ & $E_{7}$ & $c_{1}$ & $c_{2}$ & $c_{3}$ & $c_{4}$ & $c_{5}$ & $c_{6}$ & $c_{7}$ \\
\hline $\mathrm{K}^{+}$ & $2.451_{(1)}^{[+5]}$ & $3.504_{(1)}^{[+5]}$ & $4.094_{(2)}^{[+5]}$ & $4.766_{(2)}^{[+5]}$ & $\ldots$ & $\ldots$ & $\ldots$ & $6.292_{(1)}^{[-4]}$ & $2.350_{(1)}^{[-3]}$ & $1.165_{(1)}^{[-2]}$ & $2.470_{(1)}^{[-3]}$ & $\ldots$ & $\ldots$ & $\ldots$ \\
\hline $\mathrm{Ca}^{2+}$ & $2.282_{(4)}^{[+5]}$ & $3.682_{(1)}^{[+5]}$ & $4.479_{(4)}^{[+5]}$ & $\ldots$ & $\ldots$ & $\ldots$ & $\ldots$ & $3.843_{(2)}^{[-4]}$ & $8.040_{(3)}^{[-3]}$ & $8.670_{(5)}^{[-3]}$ & $\ldots$ & $\ldots$ & $\ldots$ & $\ldots$ \\
\hline $\mathrm{Sc}^{3+}$ & $1.168_{(1)}^{[+5]}$ & $2.188_{(2)}^{[+5]}$ & $4.771_{(3)}^{[+5]}$ & $6.814_{(1)}^{[+5]}$ & $\cdots$ & $\ldots$ & $\cdots$ & $9.817_{(2)}^{[-5]}$ & $6.241_{(1)}^{[-4]}$ & $2.459_{(2)}^{[-2]}$ & $6.990_{(5)}^{[-3]}$ & $\cdots$ & $\ldots$ & $\cdots$ \\
\hline $\mathrm{Ti}^{4+}$ & $1.5603_{(2)}^{[+1]}$ & $9.8857_{(1)}^{[+1]}$ & $3.7145_{(3)}^{[+2]}$ & $1.4089_{(3)}^{[+3]}$ & $5.9207_{(1)}^{[+3]}$ & $4.1099_{(6)}^{[+4]}$ & $5.1296_{(1)}^{[+5]}$ & $1.006_{(7)}^{[-6]}$ & $1.2449_{(1)}^{[-6]}$ & $4.3180_{(1)}^{[-6]}$ & $1.8210_{(7)}^{[-5]}$ & $7.9042_{(5)}^{[-5]}$ & $6.040_{(1)}^{[-4]}$ & $8.457_{(2)}^{[-2]}$ \\
\hline $\mathrm{V}^{5+}$ & $1.655_{(1)}^{[+4]}$ & $4.414_{(2)}^{[+4]}$ & $1.088_{(5)}^{[+5]}$ & $2.299_{(1)}^{[+5]}$ & $4.076_{(1)}^{[+5]}$ & $6.415_{(1)}^{[+5]}$ & $7.743_{(2)}^{[+5]}$ & $1.968_{(1)}^{[-6]}$ & $1.996_{(2)}^{[-5]}$ & $3.053_{(4)}^{[-4]}$ & $2.020_{(5)}^{[-3]}$ & $1.877_{(2)}^{[-2]}$ & $1.031_{(4)}^{[-1]}$ & $1.569_{(3)}^{[-2]}$ \\
\hline $\mathrm{Cr}^{6+}$ & $5.649_{(3)}^{[+3]}$ & $1.664_{(5)}^{[++4]}$ & $5.521_{(1)}^{[+4]}$ & $1.263_{(4)}^{[+5]}$ & $2.463_{(5)}^{[+5]}$ & $5.499_{(3)}^{[+5]}$ & $7.764_{(5)}^{[+5]}$ & $3.022_{(3)}^{[-6]}$ & $2.606_{(1)}^{[-5]}$ & $4.024_{(3)}^{[-4]}$ & $2.740_{(5)}^{[-3]}$ & $1.102_{(1)}^{[-2]}$ & $5.602_{(1)}^{[-2]}$ & $1.199_{(6)}^{[-1]}$ \\
\hline $\mathrm{Mn}^{7+}$ & $3.284_{(2)}^{[+3]}$ & $1.111_{(4)}^{[+4]}$ & $2.818_{(1)}^{[+4]}$ & $7.017_{(2)}^{[+4]}$ & $1.681_{(3)}^{[+5]}$ & $4.343_{(1)}^{[+5]}$ & $8.056_{(4)}^{[+5]}$ & $4.596_{(4)}^{[-5]}$ & $1.499_{(2)}^{[-4]}$ & $1.960_{(3)}^{[-3]}$ & $5.060_{(5)}^{[-3]}$ & $7.040_{(5)}^{[-3]}$ & $3.421_{(1)}^{[-2]}$ & $1.963_{(4)}^{[-1]}$ \\
\hline $\mathrm{Fe}^{8+}$ & $2.021_{(3)}^{[+3]}$ & $2.187_{(1)}^{[+3]}$ & $7.063_{(4)}^{[+3]}$ & $2.074_{(1)}^{[+4]}$ & $4.974_{(2)}^{[+4]}$ & $2.743_{(4)}^{[+5]}$ & $8.348_{(1)}^{[+5]}$ & $3.786_{(4)}^{[-4]}$ & $1.494_{(4)}^{[-4]}$ & $9.447_{(1)}^{[-4]}$ & $2.100_{(5)}^{[-3]}$ & $3.520_{(5)}^{[-3]}$ & $2.785_{(3)}^{[-2]}$ & $2.734_{(5)}^{[-1]}$ \\
\hline $\mathrm{Co}^{9+}$ & $1.277_{(2)}^{[+3]}$ & $9.012_{(4)}^{[+3]}$ & $2.895_{(1)}^{[+4]}$ & $9.202_{(2)}^{[+4]}$ & $1.782_{(3)}^{[+5]}$ & $4.485_{(4)}^{[+5]}$ & $9.033_{(2)}^{[+5]}$ & $1.549_{(6)}^{[-5]}$ & $2.685_{(1)}^{[-4]}$ & $4.341_{(3)}^{[-4]}$ & $3.670_{(3)}^{[-3]}$ & $1.555_{(2)}^{[-2]}$ & $5.304_{(1)}^{[-2]}$ & $2.857_{(3)}^{[-1]}$ \\
\hline $\mathrm{Ni}^{10+}$ & $1.929_{(2)}^{[+3]}$ & $9.502_{(1)}^{[+3]}$ & $2.690_{(2)}^{[+4]}$ & $6.006_{(2)}^{[+4]}$ & $1.438_{(2)}^{[+5]}$ & $4.627_{(1)}^{[+5]}$ & $1.020_{(2)}^{[+6]}$ & $1.715_{(1)}^{[-4]}$ & $5.967_{(4)}^{[-4]}$ & $2.660_{(5)}^{[-3]}$ & $7.130_{(4)}^{[-3]}$ & $1.782_{(3)}^{[-2]}$ & $8.587_{(2)}^{[-2]}$ & $3.393_{(4)}^{[-1]}$ \\
\hline $\mathrm{Cu}^{11+}$ & $4.706_{(2)}^{[+3]}$ & $1.541_{(2)}^{[+4]}$ & $2.982_{(1)}^{[+4]}$ & $7.313_{(2)}^{[+4]}$ & $2.166_{(2)}^{[+5]}$ & $6.000_{(4)}^{[+5]}$ & $1.160_{(1)}^{[+6]}$ & $5.843_{(3)}^{[-4]}$ & $1.690_{(5)}^{[-3]}$ & $6.440_{(3)}^{[-3]}$ & $1.388_{(1)}^{[-2]}$ & $2.954_{(1)}^{[-2]}$ & $1.494_{(4)}^{[-1]}$ & $3.518_{(2)}^{[-1]}$ \\
\hline $\mathrm{Zn}^{12+}$ & $1.182_{(3)}^{[+3]}$ & $5.335_{(2)}^{[+3]}$ & $1.502_{(1)}^{[+4]}$ & $4.288_{(3)}^{[+4]}$ & $1.626_{(1)}^{[+5]}$ & $4.313_{(1)}^{[+5]}$ & $1.034_{(3)}^{[+6]}$ & $9.081_{(1)}^{[-5]}$ & $1.299_{(5)}^{[-4]}$ & $1.492_{(5)}^{[-4]}$ & $3.050_{(5)}^{[-3]}$ & $3.209_{(1)}^{[-2]}$ & $1.049_{(1)}^{[-1]}$ & $4.396_{(1)}^{[-1]}$ \\
\hline
\end{tabular}

Table 9. Fit coefficients for total ground state RR rate coefficients of recombining ions, see Eq. (6), where $v_{(u)}^{[ \pm p]}$ denotes $v(u) \times 10^{ \pm p}$ with uncertainties given in lower parentheses.

\begin{tabular}{|c|c|c|c|c|c|c|}
\hline $\operatorname{Ion}^{a}$ & $\begin{array}{c}A \\
\left(10^{-11} \mathrm{~cm}^{3} \mathrm{~s}^{-1}\right)\end{array}$ & $B$ & $\begin{array}{l}T_{0} \\
(\mathrm{~K})\end{array}$ & $\begin{array}{l}T_{1} \\
(\mathrm{~K})\end{array}$ & C & $\begin{array}{l}T_{2} \\
(\mathrm{~K})\end{array}$ \\
\hline $\mathrm{K}^{+}$ & $4.528_{(3)}^{[+0]}$ & $4.234_{(2)}^{[-1]}$ & $5.931_{(3)}^{[+0]}$ & $2.897_{(3)}^{[+9]}$ & $3.049_{(4)}^{[-1]}$ & $1.645_{(3)}^{[+5]}$ \\
\hline $\mathrm{Ca}^{2+}$ & $2.248_{(4)}^{[+1]}$ & $6.605_{(1)}^{[-1]}$ & $6.175_{(1)}^{[+0]}$ & $6.032_{(1)}^{[+6]}$ & $3.158_{(2)}^{[-1]}$ & $2.100_{(2)}^{[+5]}$ \\
\hline $\mathrm{Sc}^{3+}$ & $4.196_{(2)}^{[+0]}$ & $2.250_{(1)}^{[-1]}$ & $4.232_{(1)}^{[+2]}$ & $1.110_{(1)}^{[+8]}$ & $3.993_{(1)}^{[-1]}$ & $3.973_{(3)}^{[+5]}$ \\
\hline $\mathrm{Ti}^{4+}$ & $3.989_{(3)}^{[+1]}$ & $5.658_{(2)}^{[-1]}$ & $9.517_{(3)}^{[+1]}$ & $4.776_{(2)}^{[+7]}$ & $1.065_{(4)}^{[-1]}$ & $3.534_{(2)}^{[+5]}$ \\
\hline $\mathrm{V}^{5+}$ & $4.193^{[+1]}$ & $5.887^{[-1]}$ & $1.993^{[+2]}$ & $2.207^{[+7]}$ & $9.247^{[-2]}$ & $2.012_{([)}^{[+5]}$ \\
\hline $\mathrm{Cr}^{6+}$ & $5.744_{(8)}^{[+1]}$ & $6.220_{(1)}^{[-1]}$ & $2.282^{[+2]}$ & $2.250_{(8)}^{[+7]}$ & $4.925_{(1)}^{[-2]}$ & $2.948_{[7]}^{[+5]}$ \\
\hline $\mathrm{Mn}^{7+}$ & $2.976_{(5)}^{[+1]}$ & $4.838_{(8)}^{[-1]}$ & $1.397_{(3)}^{[+3]}$ & $4.566_{(8)}^{[+7]}$ & $5.863_{(1)}^{[-2]}$ & $1.672_{(3)}^{[+4]}$ \\
\hline $\mathrm{Fe}^{8+}$ & $3.341_{(3)}^{[+1]}$ & $4.865_{(7)}^{[-1]}$ & $1.891_{(7)}^{1+3]}$ & $5.181_{(7)}^{1+7]}$ & $5.747_{(1)}^{(1)}$ & $2.734_{(9)}^{[+4]}$ \\
\hline $\mathrm{Co}^{9+}$ & $2.542_{(3)}^{[+1]}$ & $3.600_{(6)}^{[-1]}$ & $5.026_{(4)}^{[+3]}$ & $5.480_{(1)}^{[+7]}$ & $1.142_{(3)}^{[-1]}$ & $2.509_{(1)}^{[+4]}$ \\
\hline $\mathrm{Ni}^{10+}$ & $3.076_{(2)}^{[+1]}$ & $3.849_{(1)}^{(6)}$ & $5.284_{(4)}^{[+3]}$ & $4.945_{(1)}^{[+7]}$ & $1.021_{(1)}^{[-1]}$ & $2.571_{(1)}^{[+4]}$ \\
\hline $\mathrm{Cu}^{11+}$ & $3.527_{(4)}^{[+1]}$ & $4.052_{(3)}^{[-1]}$ & $5.855_{(1)}^{[+3]}$ & $5.691_{(1)}^{1+7]}$ & $8.842_{(1)}^{[-2]}$ & $3.626_{(1)}^{[1+4]}$ \\
\hline $\mathrm{Zn}^{12+}$ & $3.964_{(2)}^{[+1]}$ & $4.107_{(3)}^{[-1]}$ & $6.576_{(1)}^{[+3]}$ & $5.855_{(1)}^{[+7]}$ & $8.595_{(1)}^{[-2]}$ & $3.942_{(3)}^{[1+4]}$ \\
\hline
\end{tabular}

Notes. ${ }^{(a)}$ present work: $\ell \leq 200,3 \leq n \leq 1000$. 
D. Nikolić et al.: Dielectronic recombination of argon-like ions
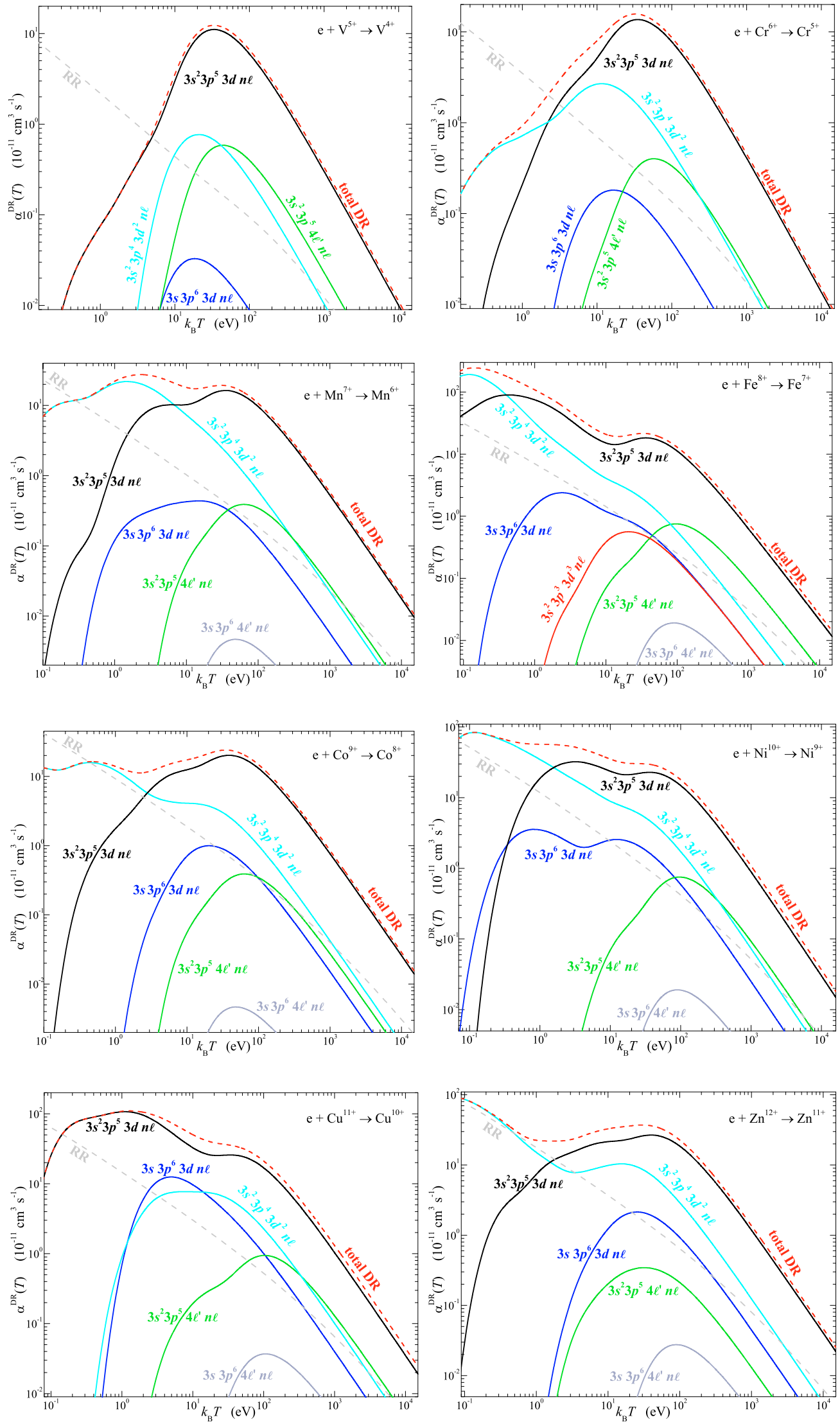

Fig. 9. Present Maxwellian-averaged DR and RR rate coefficients for V VI - Zn XIII ions. The red and gray dashed curves are the total DR and RR rate coefficients, respectfully. The intra-shell $\Delta n_{c}=0$ contributions are due to $3 \mathrm{p} \rightarrow 3 \mathrm{~d}$ (solid black curve), $3 \mathrm{~s} \rightarrow 3 \mathrm{~d}$ (solid blue curve), and $3 \mathrm{p}^{2} \rightarrow 3 \mathrm{~d}^{2}$ (solid cyan curve) core excitations; the inter-shell $\Delta n_{\mathrm{c}}=1$ contributions are due to $3 \mathrm{p} \rightarrow 4 \ell^{\prime}$ (solid green curve) and $3 \mathrm{~s} \rightarrow 4 \ell^{\prime}$ (solid gray curve) promotions. 
crude parameterization formulas that are not as reliable as our computed MCBP rate coefficients. The results reported here serve as what we assess to be the most accurate and complete set of Ar-like DR data for use in spectral diagnostic studies of laboratory and astrophysical plasmas.

Acknowledgements. This work was funded in part by NASA APRA, NASA SHP SR\&T, and PPARC grants.

\section{References}

Adelman, S. J., Gulliver, A. F., \& Lodén, L. O. 2000, A\&A, 353, 335

Aggarwal, K. M., \& Keenan, F. P. 2007, A\&A, 475, 393

Aggarwal, K. M., \& Keenan, F. P. 2008, Eur. Phys. J. D, 46, 205

Aggarwal, K. M., Keenan, F. P., Kato, T., \& Murakami, I. 2006, A\&A, 460, 331

Ansari, S. M. R., Elwert, G., \& Mücklich, P. 1970, Z. Naturforsch., 25a, 1781

Arnaud, M., \& Raymond, J. 1992, ApJ, 398, 394

Arnaud, M., \& Rothenflug, R. 1985, A\&AS, 60, 425

Badnell, N. R. 1986, J. Phys. B, 19, 3827

Badnell, N. R. 1997, J. Phys. B, 30, 1

Badnell, N. R. 2006, ApJ, 651, L73

Badnell, N. R., O’Mullane, M. G., Summers, H. P., et al. 2003, A\&A, 406, 1151

Baluja, K. L. 1986, J. Phys. B, 19, L551

Bryans, P., Badnell, N. R., Gorczyca, T. W., et al. 2006, ApJS, 167, 343

Bryans, P., Landi, E., \& Savin, D. W. 2008, ArXiv e-prints, 805

Bryans, P., Kreckel, H., Roueff, E., Wakelam, V., \& Savin, D. W. 2009a, ApJ, 694, 286

Bryans, P., Landi, E., \& Savin, D. W. 2009b, ApJ, 691, 1540

Burgess, A. 1965, ApJ, 141, 1588

Burgess, A., Manson, H. E., \& Tully, J. A. 1989, A\&A, 217, 319

Chakravorty, S., Kembhavi, A. K., Elvis, M., Ferland, G., \& Badnell, N. R. 2008, MNRAS, 384, L24

Chakravorty, S., Kembhavi, A. K., Elvis, M., \& Ferland, G. 2009, MNRAS, 393, 83

Churazov, E., Forman, W., Jones, C., Sunyaev, R., \& Böhringer, H. 2004, MNRAS, 347, 29

del Peloso, E. F., Cunha, K., da Silva, L., \& Porto de Mello, G. F. 2005, A\&A, 441, 1149

Dere, K. P., Landi, E., Young, P. R., et al. 2009, A\&A, 498, 915

Ellison, S. L., Ryan, S. G., \& Prochaska, J. X. 2001, MNRAS, 326, 628

Fawcett, B. C. 1991, Z. Phys. D. Suppl., 21, 1

Ferland, G. J., Korista, K. T., Verner, D. A., et al. 1998, PASP, 110, 761

Froese-Fischer, C., Tachiev, G., \& Irimia, A. 2006, Atomic Data and Nuclear Data Tables, 92, 607

Ghosh, T. K., \& Mukherjee, P. K. 1997, Phys. Scr., 55, 273

Gu, M. F. 2004, The Flexible Atomic Code, Atomic Processes In Plasmas: 14th APS Topical Conference on Atomic Processes in Plasmas (Santa Fe, New Mexico, USA: AIP), 730, 127

Hahn, Y. 1989, J. Quant. Spec. Radiat. Transf., 41, 315

Hahn, Y. 1991, Phys. Scr., T37, 53
Hillebrandt, W., \& Niemeyer, J. C. 2000, ARA\&A, 38, 191

Jacobs, V. L., \& Davis, J. 1983, NRL Memorandum Report, 5105, 1 Jacobs, V. L., Davis, J., Kepple, P. C., \& Blaha, M. 1977, ApJ, 211, 605

Kallman, T. R., \& Palmeri, P. 2007, Reviews of Modern Physics, 79, 79

Karrer, R., Bochsler, P., Giammanco, C., et al. 2007, Space Sci. Rev., 130, 317

Kato, T., \& Asano, E. 1999, NIFS-DATA, 54, data from NIFS DATABASE at https://dbshino.nifs.ac.jp

Kraemer, S. B., Ferland, G. J., \& Gabel, J. R. 2004, ApJ, 604, 556

Landini, M., \& Fossi, B. C. M. 1991, A\&ASS, 91, 183

Liedhal, D. A. 2000, in Atomic Data Needs for X-ray Astronomy, ed. M. A. Bautista, A. K. Kallman, \& T. R. Pradhan, Laboratory for High Energy Astrophysics (NASA Goddard Space Flight Center: HEASARC), 151

Loginov, A. V., \& Tuchkin, V. I. 2002, Opt. Spectrosc. , 93, 186

Lukić, D. V., Schnell, M., Savin, D. W., et al. 2007, ApJ, 664, 1244

Mattioli, M., Fournier, K. B., Coffey, I., et al. 2004, J. Phys. B, 37, 13

Mazzitelli, G., \& Mattioli, M. 2002, At. Data Nucl. Data Tables, 82, 313

Mazzotta, P., Mazzitelli, G., Colafrancesco, S., \& Vittorio, N. 1998, A\&AS, 133, 403

Mewe, R., Schrijver, J., \& Sylwester, J. 1980, A\&AS, 40, 323

Mewe, R., Gronenschild, E. H. B. M., \& van den Oord, G. H. J. 1985, A\&AS, 62, 197

Netzer, H. 2004, ApJ, 604, 551

Nikolić, D., Gorczyca, T. W., Fu, J., Savin, D. W., \& Badnell, N. R. 2007, Nucl. Instrum. Methods B, 261, 145

Nikolić, D., Gorczyca, T. W., \& Badnell, N. R. 2009, Phys. Rev. A, 79, 012703

Nikolić, D., Gorczyca, T. W., \& Badnell, N. R. 2010, Phys. Rev. A, 81, 030501(R)

Pettersen, K., Ekberg, J., Martinson, I., \& Reader, J. 2007, Phys. Scripta, 75, 702 Pindzola, M. S., Griffin, D. C., Bottcher, C., Buie, M. J., \& Gregory, D. C. 1991, Phys. Scr., T37, 35

Pindzola, M. S., Badnell, N. R., \& Griffin, D. C. 1992, Phys. Rev. A, 46, 5725

Savin, D. W., \& Laming, J. M. 2002, ApJ, 566, 1166

Schippers, S., Bartsch, T., Brandau, C., et al. 1998, J. Phys. B, 31, 4873

Schippers, S., Kieslich, S., Müller, A., et al. 2002, Phys. Rev. A, 65, 042723

Schmidt, E. W., Schippers, S., Bernhardt, D., et al. 2008, A\&A, 492, 265

Seon, K. I., Nam, U. W., \& Park, I. H. 2003, J. Phys. B, 36, 2679

Shirai, T., Sugar, J., Musgrove, A., \& Wiese, W. L. 2000, Spectral Data for Highly Ionized Atoms: Ti, V, Cr, Mn, Fe, Co, Ni, Cu, Kr, and Mo, J. Phys. Chem. Ref. Data, Monograph No. 8 (Melville, NY: AIP Press), http:// physics.nist.gov/PhysRefData/ASD

Smith, R. K., Brickhouse, N., Liedahl, D. A., \& Raymond, J. C. 2001, ApJ, 556, L91

Storey, P. J., Zeippen, C. J., \& Le Dourneuf, M. 2002, A\&A, 394, 753

Sugar, J., \& Corliss, C. 1985, J. Phys. Chem. Ref. Data, 14, 1, (Suppl. 2) http://physics.nist.gov/PhysRefData/ASD

TFR Group. 1980, Plasma Phys., 22, 851

Verma, N., Jha, A. K. S., \& Mohan, M. 2006, ApJS, 164, 297

Verma, N., Jha, A. K. S., \& Mohan, M. 2007, Eur. Phys. J. D, 42, 235

Verner, D. A., \& Ferland, G. J. 1996, ApJS, 103, 467

Werner, N., Durret, F., Ohashi, T., Schindler, S., \& Wiersma, R. P. C. 2008, Space Sci. Rev., 134, 337

Zeng, J. L., Wang, Y. G., Zhao, G., \& Yuan, J. M. 2006, Chin. Phys., 15, 1502 